\title{
A numerical study of two dimensional flows past a bluff body for dilute polymer solutions
}

\author{
Y.L. Xiong ${ }^{\mathrm{a}, *}$, C.H. Bruneau ${ }^{\mathrm{a}}$, H. Kellay ${ }^{\mathrm{b}}$ \\ a Univ. Bordeaux, Institut de Mathématiques de Bordeaux, INRIA Team MC2, CNRS UMR 5251, 351 cours de la Libération, 33405 Talence, France \\ ${ }^{\mathrm{b}}$ Univ. Bordeaux, Laboratoire Ondes et Matière d'Aquitaine, UMR 5798 CNRS, 351 cours de la Libération, 33405 Talence, France
}

Keywords:

Numerical simulation

Drag reduction

Dilute polymer solutions

\begin{abstract}
A B S T R A C T
In this paper, we use a simple Oldroyd B constitutive model to study the role of the viscoelasticity of dilute polymer solutions in two dimensional flows past a bluff body using numerical simulations. This investigation is motivated by the numerous experimental results obtained in quasi two dimensional systems such as soap film channels. The numerical modeling is novel for this case and therefore a com prehensive comparison is carried out to validate the present penalization method and artificial boundary conditions. In particular we focus on flow past a circular object for various values of the Reynolds number Weissenberg number, and polymer viscosity ratio. Drag enhancement and drag reduction regimes are discussed in detail along with their flow features such as the pattern of vortex shedding, the variation of lift as well as changes in pressure, elongational rates, and polymer stress profiles. A comprehensive study of the flow behavior and energy balance are carefully carried out for high Reynolds numbers. Flow instabilities in both numerical and experimental results are discussed for high Weissenberg numbers.
\end{abstract}

\section{Introduction}

Turbulent frictional drag reduction and flow structure modifica tion by polymer additives has received considerable attention since Toms's discovery in 1949 [49]. On the other hand, the effects of polymers on the drag of a bluff body are less studied. Reducing the drag of bluff bodies like ships and submarine hulls to achieve higher speeds and reduced energy cost has an equivalent industrial significance. Furthermore, the drag behavior of a bluff body in di lute polymer solutions can shed some light on the skin friction with rough boundaries [16]. For the flow past a bluff body, pressure drag is an important part of the total drag, i.e. an optimal scheme for skin friction reduction of the turbulent boundary layer may be useless when pressure drag dominates. Hence, it is reasonable to classify the problem of drag reduction for flow past a bluff body in the important problems category in fluid mechanics.

Numerical simulations of unbounded creeping flow of dilute polymer solutions past cylinders and spheres using a suspension of dumbbells model with finite extensibility were performed by Chilcott and Rallison [11]. The drag coefficient variation with the Weissenberg number for the flow past a sphere first dips slightly, and then rises for all model parameters. For the flow past a cylin der, the drag increases with Weissenberg number. The decrease of

\footnotetext{
* Corresponding author.

E-mail address: xylcfd@hotmail.com (Y.L. Xiong).
}

drag has not been found contrarily to the case of flow around a sphere. This is due to the blockage ratio [19]. In their numerical investigation, there is no drag reduction at small blockage ratio when $B<0.1$, however the trend is completely reversed for flows with stronger blockage. A recent experiment using polyethylene oxide (PEO) with molecular weight 4 million to study the flow around a cylinder in the Stokes regime was performed by Francois et al. [16]. Drag enhancement above a critical Weissenberg number for different polymer concentration solutions was observed. There is no drag reduction in their subsequent pipe flow experiment where the drag was measured for a specially designed pipe with large roughness by small cylinders on the surface of the wall. Their experimental investigations shed some light on the drag reduction obtained with polymer solutions in rough pipes reported in earlier experiments [50].

James and Acosta [23] measured the heat transfer and drag for the flow of dilute polymer solutions past a cylinder at $R e<50$. The concentration increase results in the reduction of heat transfer and enhancement of drag at a fixed Reynolds number. They suggest the importance of $R e / W i$ as an index to describe the effects of elasticity. They also used a dye injection to visualize fluid flow which showed an increase of the effective wake size, and streamline asymmetry for increasing polymer concentration. Kato and Mizuno [24] mea sured the drag force in the Re range of $15 \times 10^{4}$ for the flow past a cylinder with and without polymer. They found that drag forces in the Re range of 1100 are barely affected by the polymers. Important drag reduction was found in the $R e$ range of 
$10^{3} 5 \times 10^{4}$ for the polymer solution. The maximum drag reduc tion is barely affected by the Reynolds number in the range of $\operatorname{Re}>8 \times 10^{3}$. They also found drag reduction can be reduced at high Weissenberg number for high Reynolds number flows. From the measurement of pressure distribution around a cylinder, they found that the presence of polymers in solution may increase the pressure near the rear of the cylinder and delay the separation for the high Reynolds number flow.

In the numerical simulations of viscoelastic flows by Oliveira [39], drag reduction was found in the Re range of 50150 for the FENE CR fluid, which is in agreement with three dimensional sim ulations of flow past an ellipsoid for the FENE P fluid [32]. Drag reduction and drag enhancement found in the literature for various Reynolds numbers are listed in Table 1. Oliveira also pointed out that the vortex shedding frequency is changed by elasticity, and that the formation length of the wake is increased by the polymer. These conclusions are supported by the experimental works of $\mathrm{Ca}$ dot and coworkers $[8,9]$.

A majority of studies on the flow past a cylinder focus on creep ing flow at low Reynolds number. The flow past a confined cylinder has no geometrical singularities and includes both elongational and shear deformation. Thus it is suitable to serve as a benchmark both for numerical and experimental investigations on the effects of viscoelasticity. A confined cylinder in channel flow with block age ratio $B=0.5$ had served as a benchmark and a large number of numerical and experimental studies are related to this configu ration $[3,10,11,14,15,1921,30,33,35,38,40,47]$. The drag behavior is summarized in Table 2. The results show consistently that drag first slightly decreases then increases with Wi, just like drag behav ior of flow past a sphere [22] . On the other hand, the experiments have not validated such behavior for this configuration. Indeed, drag enhancement is also found for the polymer solutions. It should be noted that the blockage ratio 0.5 is too large to separate the influence of the constraining wall and the extensional flow near the stagnation point, and may cause severe three dimensional effects as suggested by experimental results [51].

Furthermore, the elastic instability is now becoming a more and more important topic for the flow of polymer solution $[17,18]$. McKinley et al. [33] demonstrated that the instability is generated in the wake near the rear of stagnation point then convected downstream. They found that the steady planar stagnation flow in the downstream wake becomes a three dimensional cellular structure when the Weissenberg number exceeds a critical value. Shiang et al. [45] performing experiments for viscoelastic flow past a confined cylinder showed similar behavior in which the visco elastic flow bifurcates from steady two dimensional flow to steady three dimensional flow for values of Weissenberg number greater than a critical value. In two dimensional numerical simulations, a periodical bubble at the rear of the cylinder was found, this also in duces a periodical drag force for the viscoelastic creeping flow past a cylinder in these simulation results [38]. On the flow instability side, Cadot and Kumar [8] suggested that viscoelasticity delayed the development of two dimensional instability by injecting poly mer solutions into a cylinder wake. Sahin and Owens [44] also

Table 1

Literature review on drag behavior for the viscoelastic fluid past a cylinder.

\begin{tabular}{llll}
\hline Author & Method & Re range & drag \\
\hline James and Acosta [23] & Experiment & $1-50$ & Enhancement \\
Kato and Mizuno [24] & Experiment & $1000-50,000$ & Reduction \\
Chilcott and Rallison [11] & FD & $<1$ & Enhancement \\
Huang and Feng [19] & FEM & $0.1-10$ & Enhancement \\
Oliveira [39] & FVM & $50-150$ & Reduction \\
Cressman et al. [13] & Experiment & $\mathrm{O}(100)$ & - \\
Francois et al. [16] & Experiment & $\ll 50$ & Enhancement \\
\hline
\end{tabular}

indicated the critical Reynolds number for the onset of vortex shedding in the viscoelastic fluid is higher than in the Newtonian case by direct numerical simulation (DNS) and linear stability anal ysis. This conclusion is supported by the experimental work of Pipe and Monkewtiz [41]. They found that the critical Reynolds number increased for the low concentrations of PEO solutions. Their laser doppler anemometry (LDA) measurements have shown that the lateral velocity fluctuations on the wake centerline are similar to the Newtonian case at low concentrations although a reduction in strength is noted at their highest concentration. They suggest that the normal stress differences and the elongational viscosity play a role in the development of the instability. More recent three dimensional simulations of flows past a cylinder [42,43] have also demonstrated that polymers suppress the three dimensional New tonian mode B instability, and even eliminate all three dimen sional flow structure for high polymer extensibility.

In this paper, and while most studies cited above examine three dimensional situations, we rather focus on the two dimensional case. Our choice is motivated by recent experiments using soap films and which have shown that the effects of viscoelasticity are nontrivial $[1,13,25,27]$. In earlier work, we have already shown that the numerical scheme we will use for this study captures the main features of such quasi two dimensional flows both for the Newto nian and non Newtonian cases [5,6,25 29,46,53,54]. Here we use two dimensional DNS to study the drag and flow behavior of dilute polymer solutions in an adequate parameter space of $R e$ Wi. The dilute polymer solutions are treated as Boger fluids using the Old royd $B$ model. Despite the limitations of such a model, we have found it to capture the main features of such flows in previous work $[53,54]$. We focus on flows past a circular bluff body, which is widely studied in Newtonian fluids [52], and investigate the flow behavior for small blockage ratios allowing for a broad range of both Reynolds numbers and Weissenberg numbers to understand the effect of viscoelasticity on the flow and the drag behavior. This paper is organized as follows: After describing the numerical mod el and its validation, the effect of polymer viscosity ratio is studied. Then the drag and flow behavior is described in detail and finally some instabilities caused by the presence of polymers are discussed.

\section{Governing equations, boundary conditions and numerical methods}

The present study is the flow past a single cylinder which is placed symmetrically in a plane channel with unit width. The spa tial computational domain $\Omega$ is illustrated in Fig. 1 . The governing equations of a non dimensional unsteady incompressible flow for Oldroyd B fluid [37] in the domain $\Omega \times(0, T)$ read:

$$
\left\{\begin{array}{l}
\boldsymbol{\nabla} \cdot \mathbf{u} \quad 0 \\
\partial_{t} \mathbf{u}+(\mathbf{u} \cdot \nabla) \mathbf{u}+\frac{\mathbf{u}}{K} \quad \boldsymbol{\nabla p}+\frac{1}{R e}\left[\left(\begin{array}{ll}
1 & \epsilon) \Delta \mathbf{u}+\frac{\epsilon}{W i} \nabla \cdot \boldsymbol{\sigma}
\end{array}\right]\right. \\
\partial_{t} \boldsymbol{\sigma}+(\mathbf{u} \cdot \nabla) \boldsymbol{\sigma}+\frac{\boldsymbol{\sigma}}{K}+\frac{\boldsymbol{\sigma} \mathbf{I}}{W i} \quad(\nabla \mathbf{u}) \cdot \boldsymbol{\sigma}+\boldsymbol{\sigma} \cdot(\boldsymbol{\nabla} \mathbf{u})^{T}+\kappa \Delta \boldsymbol{\sigma}
\end{array}\right.
$$

where $\mathbf{u}(\mathbf{x}, t)=(u(\mathbf{x}, t), v(\mathbf{x}, t))$ is the velocity vector, $p(\mathbf{x}, t)$ is the local non dimensional pressure, and $\mathbf{x}=(x, y)$ represents the space vector in Cartesian coordinates. $\boldsymbol{\sigma}$ is a symmetrical conformation tensor of the polymer molecular that gives three additional unknowns in two dimensions $\sigma_{x x}, \sigma_{x y}, \sigma_{y y}, \epsilon$ is the zero shear contribution of poly mers to the total polymer solution viscosity. Setting $\epsilon=0$ restore the Navier Stokes equations for a Newtonian fluid. In order to use a Cartesian mesh, the solid obstacle is taken into account by the penalization method [2]. The parameter $K$ is the nondimensional permeability coefficient used in the penalization term; it is set to $10^{16}$ in the fluid zone to recover the genuine equations and to $10^{7}$ in the solid cylinders to enforce $\mathbf{u}$ and $\boldsymbol{\sigma}$ to vanish. Penalizing the conformation tensor is not necessary, however it can make the 
Table 2

Literature review on drag behavior for viscoelastic fluid flow past a cylinder, with $B=0.5$, in the Stokes regime.

\begin{tabular}{|c|c|c|c|}
\hline Author & Method & Model & Drag Behavior \\
\hline Baaijens et al. [3] & DG & Giesekus/PTT & Reduction \\
\hline Liu et al. [30] & FEA & $\begin{array}{l}\text { Giesekus/FENE-P/ } \\
\text { CR }\end{array}$ & Reduction \\
\hline Dou et al. [14] & FVM & Oldroyd-B & Reduction \\
\hline Fan et al. [15] & FEA & Oldroyd-B & Reduction \\
\hline Owens et al. [40] & SEM & Oldroyd-B & Reduction \\
\hline Verhelst and Nieuwstadt [51] & Experiment & - & Enhancement \\
\hline Oliveira and Miranda [38] & FVM & FENE-P & Reduction \\
\hline Hulsen et al. [21] & FEA & Oldroyd-B & Reduction \\
\hline
\end{tabular}

FVM, finite volume method; FEM, finite element method; SEM, spectral element method; DG, discontinuous Galerkin.

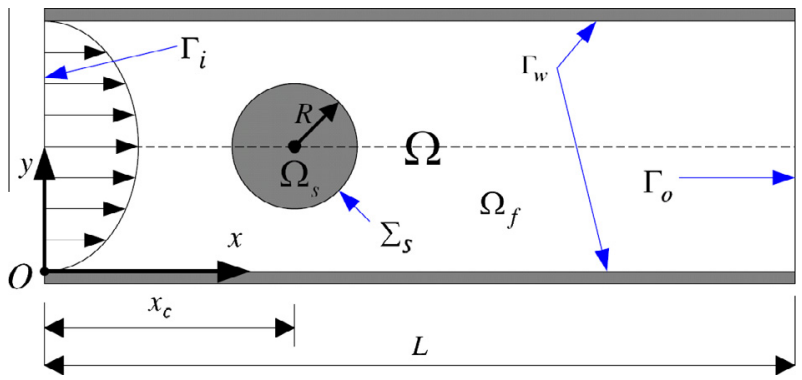

Fig. 1. Sketch of the computational domain.

numerical simulations more stable. The last term in the equations is an artificial diffusive term to prevent numerical instabilities, $\kappa$ is ta ken small enough to not affect significantly the solution in this study as shown in [48]. The Reynolds number Re and the Weiss enberg number $W i$ are non dimensionalized by the same referenced velocity and length. In our results, $R e$ and $W i$ are defined by $R e=U D /$ $\eta$ and $W i=\tau U / R$ respectively, where $U=1$ is the mean velocity of the inlet Poiseuille flow, $R$ and $D$ correspond to the radius and the diameter of the cylinders used which are located at one width from the entrance. Here $\eta$ and $\tau$ are respectively the kinematic viscosity and the relaxation time of the polymer solutions.

The boundary conditions are given as follows:

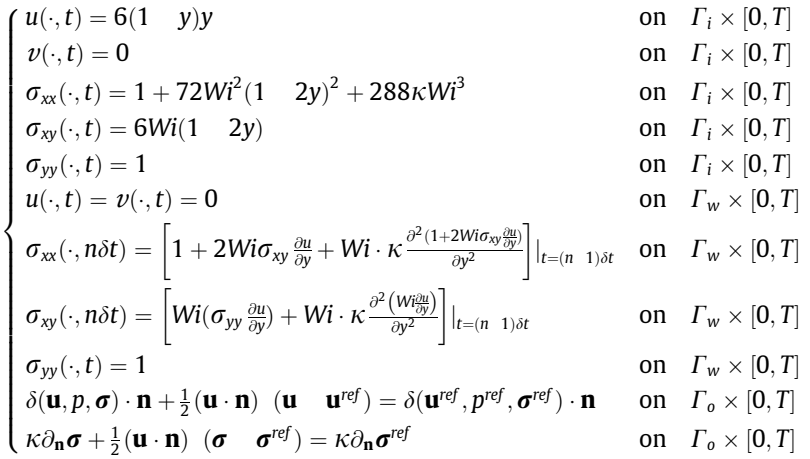

A two dimensional planar Poiseuille flow is used for the inlet velocity boundary $\Gamma_{i}$, an analytical results from constitutive equa tion are set for the tensor. The no slip condition is used on the channel wall $\Gamma_{w}$, a time splitting method is used for giving the boundary value of the tensor, where $\delta t$ is the time step of our sim ulations. An artificial non reflecting boundary condition is ob tained and put on the outlet boundary $\Gamma_{o}$ to avoid using a very long computational channel [4], where $\delta$ is the tensor in governing equations.
The approximation is performed by means of finite differences for the velocity, pressure and for the elastic stress tensor. In this method, the time interval is divided into uniform time steps $\delta t$. A second order Gear scheme is used for the time discretization. The system of equations is discretized in space on an uniform stag gered grid $G_{h}$ the mesh size of which is $h=\delta x=\delta y$, where $\delta x$ and $\delta y$ are the step discretizations in each direction. The discrete values of the pressure $p$ and the diagonal elements of the conformation ten sor $\sigma_{x x}, \sigma_{y y}$ are located at the center of each cell, the discrete values of the other element $\sigma_{x y}$ are located at the corner of the cell and those of the velocity field are located at the middle of the sides.

The discretization of the coupled velocity pressure tensor sys tem of equations allowed to solve a discrete linear system $L_{h} V_{h}^{n} \quad B_{h}^{n}{ }_{1}^{1}$ where $L_{h}$ represents the discrete operator, $B_{h}^{n} 1$ is the discrete explicit part and $V_{h}^{n} \quad\left(\mathbf{u}_{h}^{n}, p_{h}^{n}, \boldsymbol{\sigma} 2_{h}^{n+1 / 2}\right)$ is the approximate solution to compute. We have used a time splitting to first solve the coupled pressure and velocity equations then the constitutive equations. This discrete linear system is solved by the FAS (Full Approximation Scheme) V cycle multigrid solver on a $2048 \times 512$ mesh when $\Omega=(0,4) \times(0,1)$. The details of the numerical methods can be found in [7].

\section{Validation and comparison with experimental results}

\subsection{Numerical validation}

Our computations employed a sequence of grids from the fine grid Gp to the coarsest grid G1 as coarse as $16 \times 4$ cells. In order to make sure our mesh is adequate to resolve and capture with accuracy the features of the flow around the cylinder, we compared the drag coefficients and the total kinetic energy

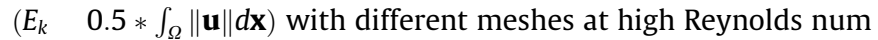
ber. The drag coefficient and the kinetic energy are listed in Table 3 and show a subtle difference between the meshes. A more efficient and obvious comparison can refer to Fig. 2, which displays the pressure and streamwise velocity profiles at the near wake of the cylinder on different meshes. From the figure, the grid G7 is insuf ficient to capture the near wake flow of the cylinder, but the grid G8 and G9 are consistent with each other even though they are not perfectly superimposed. These results show that the main fea tures can be captured on G8.

\subsection{Benchmark problem Stokes flow with blockage ratio 0.5}

The flow past a cylinder is used to study computational rheol ogy and served as a numerical benchmark problem. Numerous works on this problem have emerged [3,10,11,14,15,19 $21,30,33,35,38,40,47]$ using different viscoelastic models and com putational methods. The focus is on the drag coefficient for various Weissenberg numbers under creeping flow conditions ( $R e$ is small). The viscosity ratio of the polymer $\epsilon$ is 0.41 , the blockage ratio, i.e. the ratio of the cylinder diameter to the channel width, is $B=2 R /$ $H=0.5$.

Most of the authors adopt a very long channel, for instance $L / R$ are set to 30 [21], 36 [10], 80 [38], 28 [14] respectively. Here, we use a shorter channel with $L / R=16$. The lengths upstream and downstream of the cylinder are chosen to be $3 R$ and $11 R$. For the

Table 3

Drag coefficient and energy on different computational meshes for $R e=3000$ $W i=1.5, \epsilon=0.01$ and $B=0.1$.

\begin{tabular}{llll}
\hline & $\mathrm{G} 7(1024 \times 256)$ & $\mathrm{G} 8(2048 \times 512)$ & $\mathrm{G} 9(4096 \times 1024)$ \\
\hline$C_{d}$ & 1.81 & 1.94 & 1.97 \\
$E_{k}$ & 4.78 & 4.86 & 4.92 \\
\hline
\end{tabular}




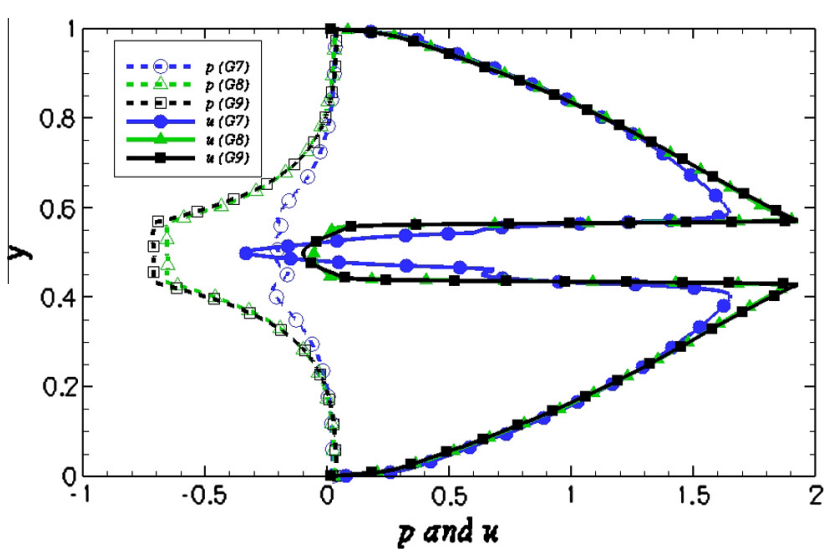

Fig. 2. The pressure and streamwise velocity profiles on different meshes at $\left(x-x_{c}\right) / R=1.5$ for $R e=3000, W i=1.5, \epsilon=0.01$ and $B=0.1$.

steady creeping flow, the advective inertial forces are small com pared to viscous forces. Hence Stokes equations, which ignore the inertial forces term of the Navier Stokes equations, are widely adopted in the references $[10,21,40]$. However, the high Reynolds number $(R e)$ flows are our interest. Therefore we use the complete momentum equation to simulate the benchmark at $R e=0.5$.

Because the inertial force is very small for the Stokes flow, the drag coefficient at this section is defined as

$$
C_{d} \frac{F_{D}}{4 \pi \eta U \rho}
$$

where $\rho$ is the density of the fluid and $F_{D}$, which is used as an index to judge whether we have obtained a steady state flow, is the drag force acting on the cylinder.

The drag coefficients of the cylinder for the Newtonian fluid are listed in Table 4 in order to compare with those of the literature. Let us note that with the penalization method the cylinder is approximated by a cloud of points without a representation of its boundary. So there is an approximation of the shape of the cylin der. Nevertheless, the results are in good agreement with the liter ature values.

The computed drag coefficients for the Oldroyd B fluid have been made dimensionless as for the Newtonian fluid and compared with published data in Table 5. All the data in the table give a sim ilar trend. The drag coefficients computed here turn out to be in very good agreement with the referenced values. It is very difficult to obtain an accurate mesh convergence result at $W i>0.7$ for the benchmark problem due to the well known high Weissenberg number instability. The maximum drag reduction for this kind of flow occurs at $W i=0.6$ with about $10 \%$ drag reduction [12].

Table 4

Drag coefficient $C_{d}$ for the Newtonian fluid with $B=0.5$ at Stokes regime.

\begin{tabular}{lllll}
\hline Author & Methods & hp & $N_{c}$ & $C_{d}$ \\
\hline Huang et al. [20] & FVM & 0.00714 & & 10.455 \\
Dou and Phan-Thien [14] & FVM & 0.040 & 156 & 10.490 \\
Oliveira and Miranda [38] & FVM & 0.0157 & 475 & 10.544 \\
Sun et al. [47] & FEM & 0.056 & & 10.532 \\
Mitsoulis [35] & FEM & & 98 & 10.529 \\
Liu et al. [30] & FEM & 0.0136 & & 10.532 \\
Caola et al. [10] & FEM & 0.002 & & 10.528 \\
Hulsen et al. [21] & FEM & 0.0148 & & 10.533 \\
Present simulations & FDM & 0.0078125 & & 10.529 \\
\hline
\end{tabular}

FVM, finite volume method; FEM, finite element method; FDM, finite difference method; hp, minimum cell spacing (normalized by $R$ ); $N_{c}$, boundary nodes on the cylinder.
Table 5

Drag coefficient variation for the Oldroyd-B fluid with $B=0.5$ in the Stokes regime (normalized by the drag of the Newtonian fluid).

\begin{tabular}{llllll}
\hline$W i$ & This work & Ref. [21] & Ref. [14] & Ref. [15] & Ref. [40] \\
\hline 0.2 & 0.95734 & 0.95670 & 0.95773 & 0.95663 & \\
0.4 & 0.91474 & 0.91114 & 0.91812 & 0.91108 & \\
0.6 & 0.89967 & 0.88995 & 0.90952 & 0.88985 & 0.88983 \\
\hline
\end{tabular}

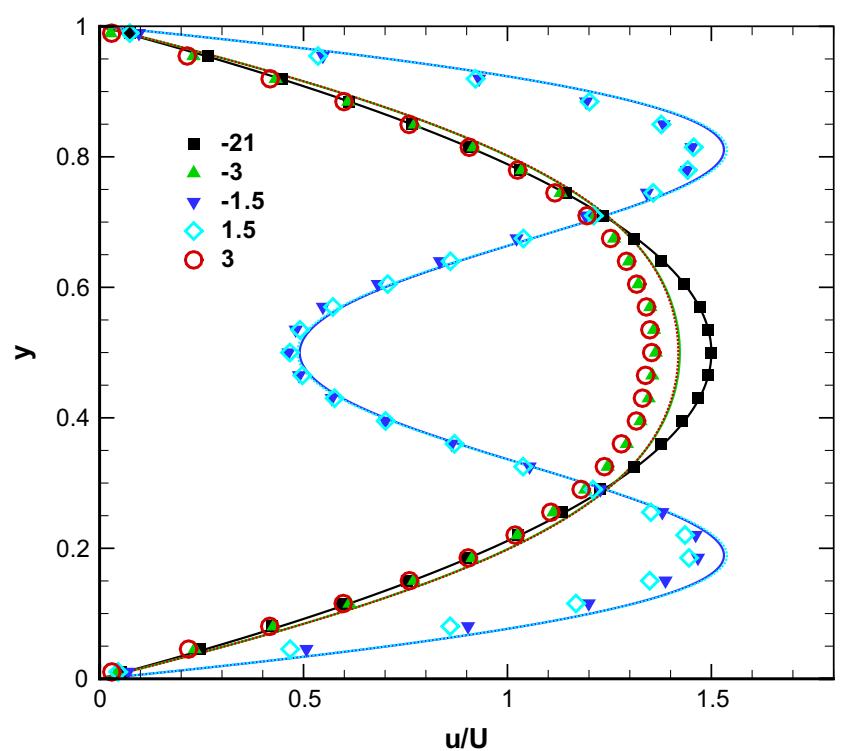

Fig. 3. Comparison of predicted velocity profiles for Newtonian fluid flow at $R e=0.307$ and $B=0.5$ with measurements by Verhelst and Nieuwstadt [51]; the numerical results (the lines) are on top of each other at \pm 1.5 and \pm 3 .

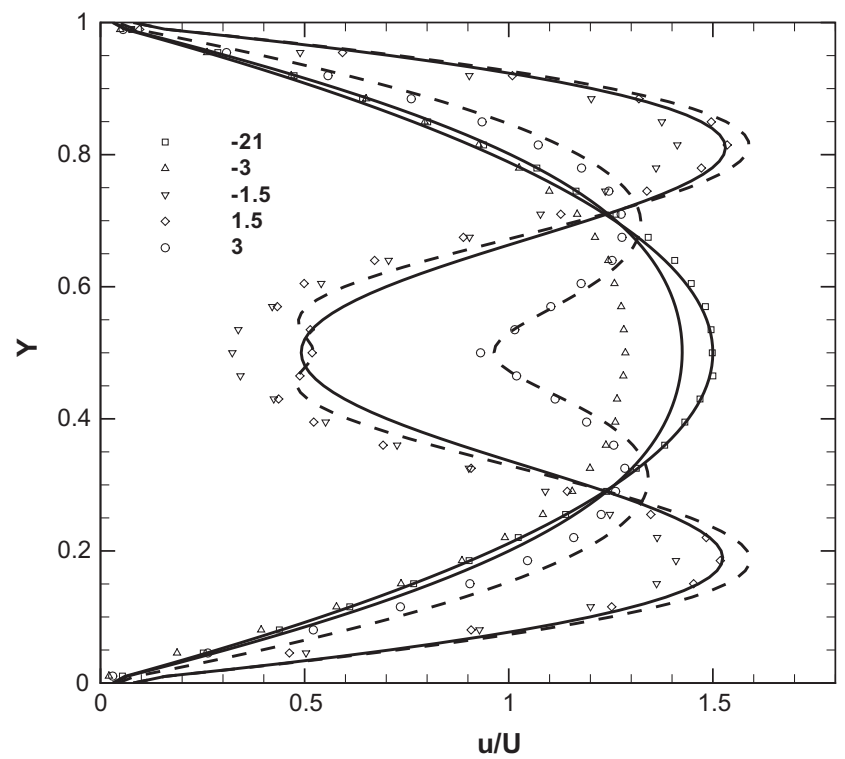

Fig. 4. Comparison of predicted velocity profiles for two-dimensional numerical simulation of Oldroyd-B fluid at $W i=1.813, R e=0.228, B=0.5$ and $\epsilon=0.01$, with three-dimensional experimental measurements by Verhelst and Nieuwstadt [51] for a polyacrylamide solution at a flow rate of $0.052 \mathrm{l} / \mathrm{s}$. (The solid lines show the numerical results upstream of the cylinder, the dashed lines represent the downstream results.) 


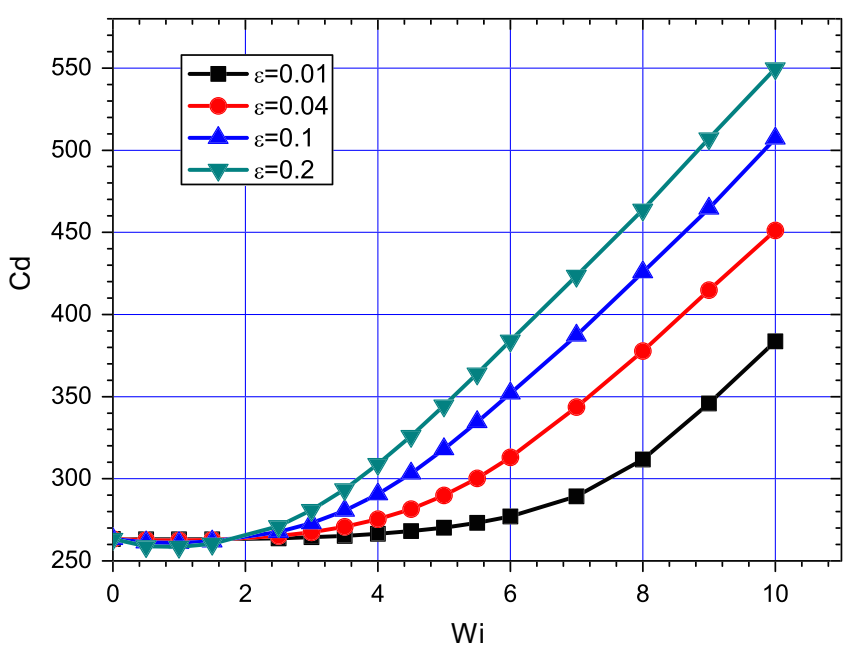

(a) $\operatorname{Re}=0.1$

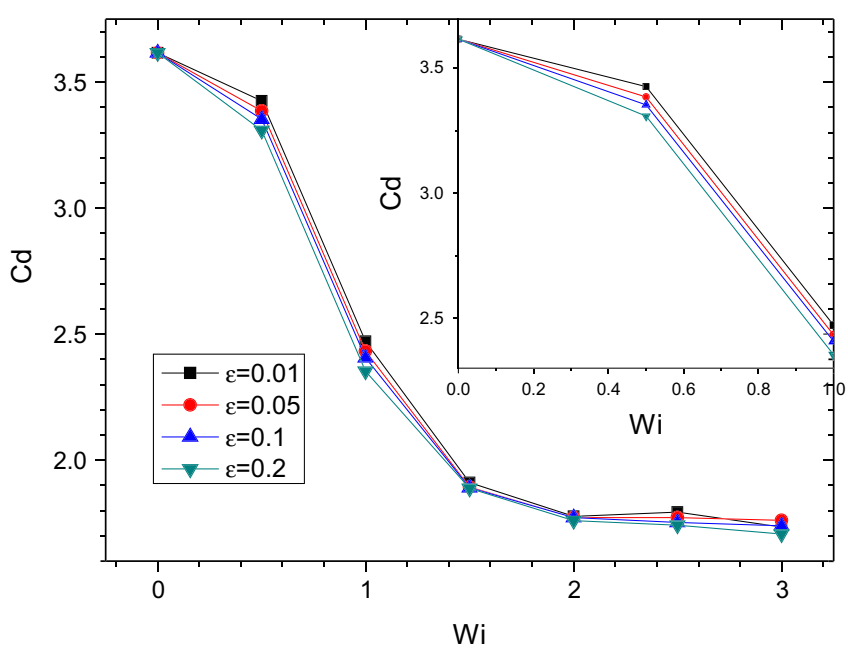

(b) $\operatorname{Re}=2000$

Fig. 5. The effects of the polymer viscosity ratio on the drag coefficient of the cylinder for $B=0.1$. Inset: Zoom for drag coefficient.

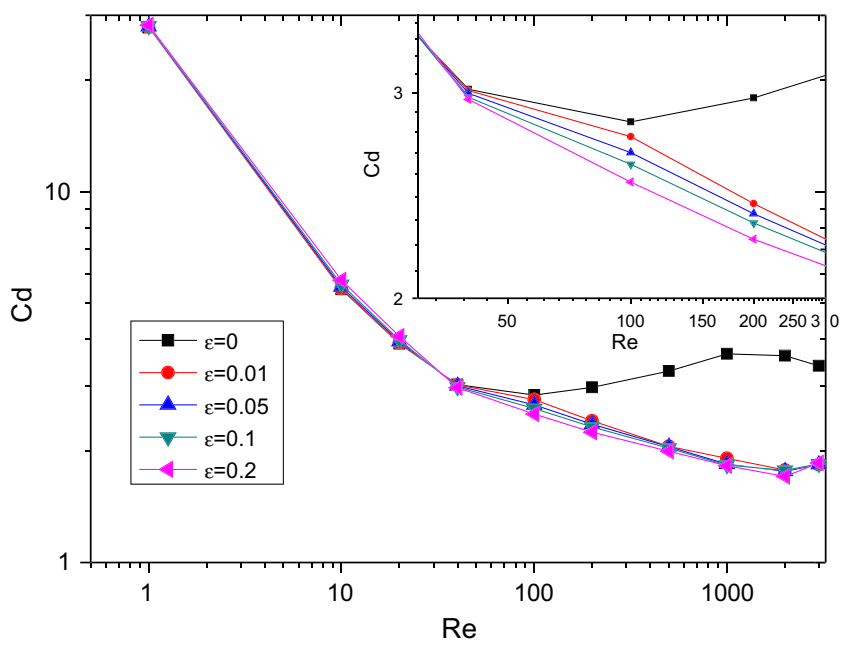

Fig. 6. The effects of the polymer viscosity ratio on the drag coefficient of the cylinder for $W i=2$ and $B=0.1$. Inset: Zoom for drag coefficient.

\subsection{Comparison with three dimensional experimental results}

Verhelst and Nieuwstadt [51] have performed experimental measurements of velocity and drag for the flow past a circular cyl inder in a rectangular channel with a width of $20 \mathrm{~mm}$ and a height of $160 \mathrm{~mm}$. They mounted a cylinder with a diameter of $10 \mathrm{~mm}$ in the symmetry plane of this channel. This configuration gives an as pect ratio $(A R)$, the ratio of the channel height to the width, $A R=8$ and a blockage ratio $B=0.5$. The section planes in the spanwise give a similar configuration to the benchmark problem. They have measured the velocity profiles of the stokes flow on the symmetry section plane. Concretely, five different locations in the streamwise direction $x$ are $\left(x \quad x_{c}\right) / R=21, \quad 3,1.5,1.5$ and 3, where $x_{c}$ is the position of the cylinder center in the streamwise coordinate and $R$ is the radius of the cylinder. Since the Reynolds number in these experiments is low, three dimensional effects are small and we can make a comparison with our two dimensional simulations.

Fig. 3 shows the comparison of the velocity profiles between the two dimensional simulation and the three dimensional experiment for the Newtonian glucose/water solution at $\left(x \quad x_{c}\right) / R=21,3$, 1.5 (upstream of the cylinder) and $\left(x \quad x_{c}\right) / R=1.5,3$ (downstream of the cylinder). For the creeping flow, the fore aft symmetry is well captured by both the experimental and numerical results (coincidence for $\left(x \quad x_{c}\right) / R= \pm 1.5, \pm 3$ ). The velocity profiles at the inlet part $\left(\begin{array}{ll}x & x_{c}\end{array}\right) / R=21$ are superimposed for the numerical and experimental results, but the profiles near the cylinder are dif ferent. The numerical results are always larger than the experi mental results. This is due to three dimensional effects and the presence of wall. A pressure difference arises between the regions of the cylinder in contact with the wall and the midplane region of the cylinder: The stagnation pressure at the symmetric plane has the largest value, larger than the value near the spanwise walls. This pressure difference induces the fluid flows toward the span wise walls.

We have not found results in the literature to discuss how vis coelastic flow affects the secondary flow. Fig. 4 shows the compar ison of predicted velocity profiles for Oldroyd B fluid at $W i=1.813$ with experimental measurements by Verhelst and Nieuwstadt [51] for a polyacrylamide solution at a flow rate of $0.052 \mathrm{l} / \mathrm{s}$. We observe that the numerical velocity is still larger than the experimental one especially for the upstream flow. The fore aft symmetry present in the Newtonian flow is lost for the polymer solutions and Oldroyd B fluid in our simulation. In this test the downstream velocity profile is modified dramatically. There is a local velocity maximum on the centerline at $\left(x \quad x_{c}\right) / R=1.5$ and a local velocity minimum on the centerline at $\left(\begin{array}{ll}x & x_{c}\end{array}\right) / R=3$ for the velocity profiles behind the cyl inder. The qualitative modifications induced by elasticity are well represented by the present two dimensional simulations.

\section{The effects of polymer viscosity ratio}

Huang and Feng [19] had reported the effects of the blockage ratio on the drag force. In their numerical investigations, there is no drag reduction at a small blockage ratio $B<0.1$, however the drag is reduced for flow with stronger blockage. Hence we mini mize the effect of the channel walls on drag by using a smaller blockage ratio $B=0.1$. All remaining calculations in this paper are for $B=0.1$.

The character of the dilute polymer solution can be described by its concentration and the characteristics of the polymer molecules. In our governing equations, the polymer solutions are character ized by the polymer viscosity ratio and the characteristic relaxa tion time. The concentration of the polymer solution can affect 


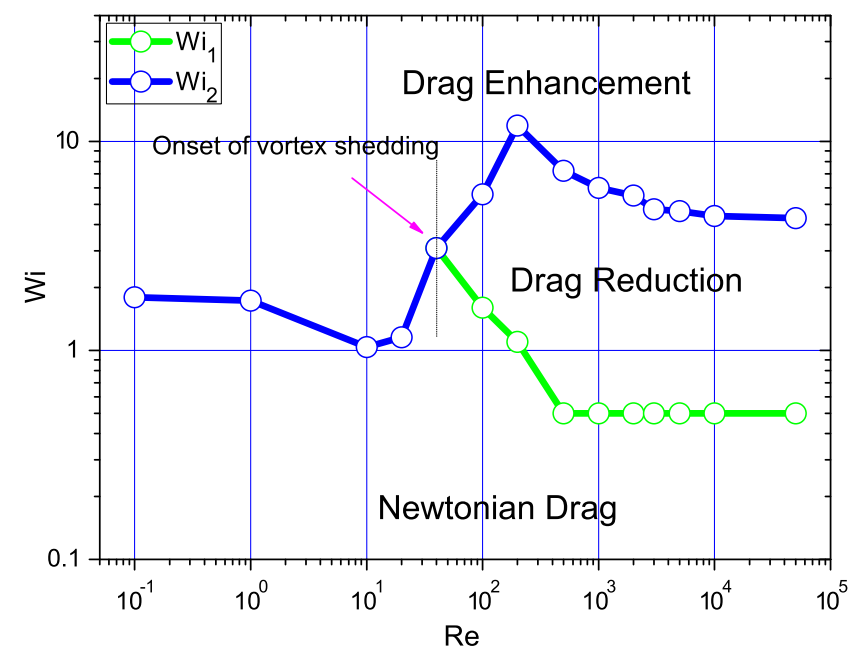

(a) The drag phase diagram

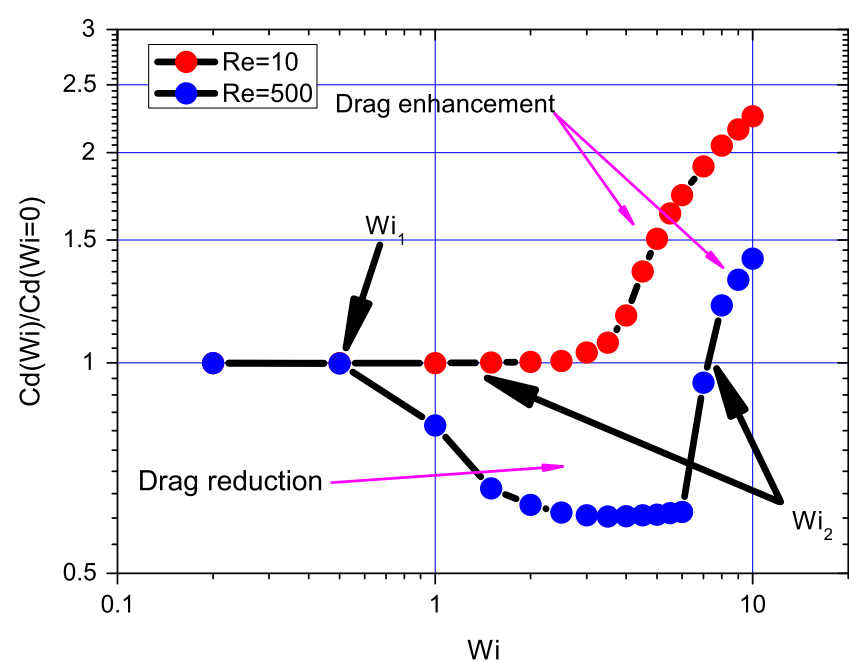

(b) The definition of $W i_{1}$ and $W i_{2}$

Fig. 7. The drag phase diagram and the definition of $W i_{1}$ and $W i_{2}$ for $\epsilon=0.01$ and $B=0.1$.

Table 6

$W i_{2}$ at different Reynolds number for $\epsilon=0.01$ and $B=0.1$.

\begin{tabular}{|c|c|c|c|c|c|c|c|c|c|c|c|c|c|}
\hline$R e$ & 0.1 & 1 & 10 & 20 & 40 & 100 & 200 & 500 & 1000 & 2000 & 3000 & 5000 & 10,000 \\
\hline$W i_{2}$ & 1.79 & 1.73 & 1.04 & 1.16 & 3.09 & 5.58 & 11.89 & 7.23 & 6.00 & 5.50 & 4.73 & 4.64 & 4.39 \\
\hline
\end{tabular}

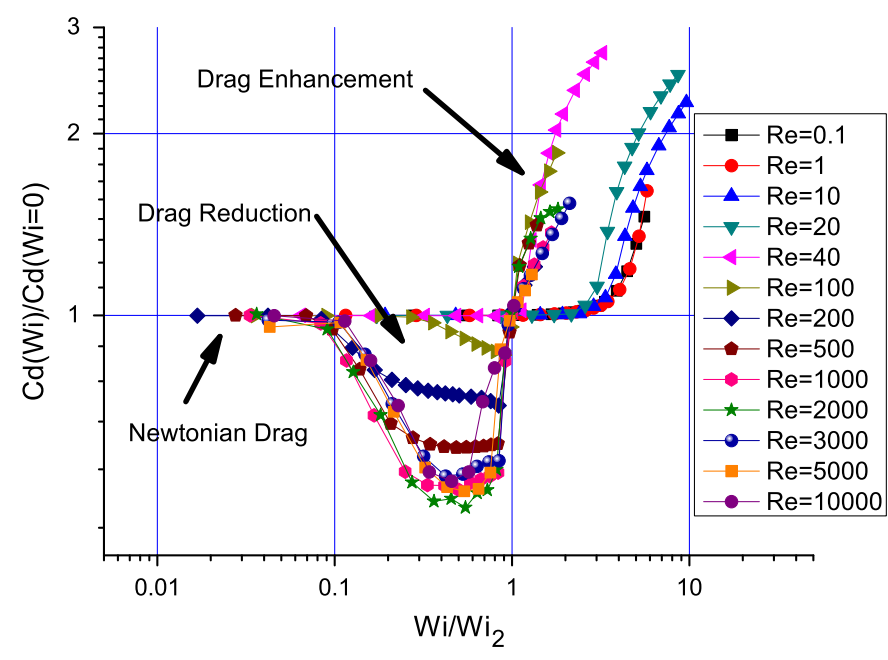

Fig. 8. The drag versus $W i / W i_{2}$ for $\epsilon=0.01$ and $B=0.1$.

both the viscosity and the relaxation time. The drag force map of the cylinder is affected by the Reynolds number $R e$, the Weiss enberg number $W i$ and the polymer viscosity ratio $\epsilon$.

In this section we will carry out a few tests for various $\epsilon$ values and a fixed Reynolds number or a fixed Weissenberg number. Using four different values of $\epsilon$ for dilute polymer solutions, we will examine the effect of the polymer viscosity ratio on the drag force of the cylinder for the selected parameter range. For the drag coefficient $C d$, we use the usual definition: $C d=F_{D} / \rho U^{2} R$, where $F_{D}$ is the drag force and $\rho$ is the density of the polymer solution.

Fig. 5 shows the drag coefficient of the cylinder for four differ ent values of $\epsilon$ versus the Weissenberg number at $R e=0.1$ and $R e=2000$. For $R e=0.1$, the variation of the drag force is very small for low Weissenberg numbers, and the drag force shows a slight minimum value at $W i=1$ by a careful observation. The drag is enhanced above $W i=2$. The drag continues to increase with

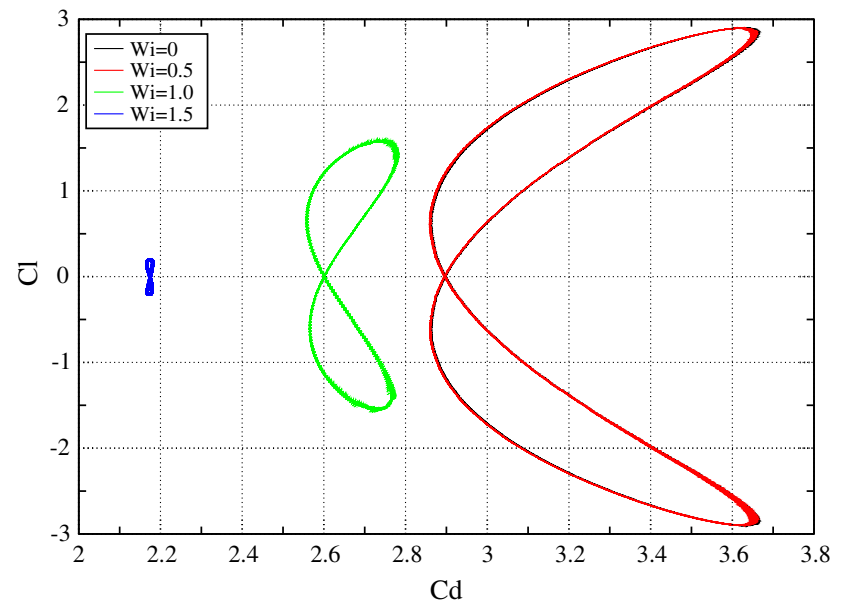

Fig. 9. Phase portrait of the drag and lift forces for $\epsilon=0.01, B=0.1$ and $R e=500$.

Weissenberg number for the different polymer viscosity ratios. The higher is the value of $\epsilon$, the more the drag deviates from the Newtonian fluid case ( $W i=0$ in the figure). For $R e=2000$, the drag coefficient decreases as the Weissenberg number increases. A tran sition is observed at low Weissenberg number according to the $\epsilon$ value; but for higher values of Weissenberg number, there is no noticeable change of the drag coefficient with a variation of $\epsilon$. For low Reynolds number, inertial force is negligible; the modi fication of the flow behavior by elastic force is comparatively smal ler than that at high Reynolds number as the extension of polymer is smaller even though an infinite extension model is used. The in crease of $\epsilon$ increases the effect of elastic force obviously. However at high Reynolds number, inertial force and elastic force dominate the flow; the extension of polymer molecule can be very high with the present Oldroyd B model. Furthermore non linear inertial force in governing equations results in the non linear effect of 


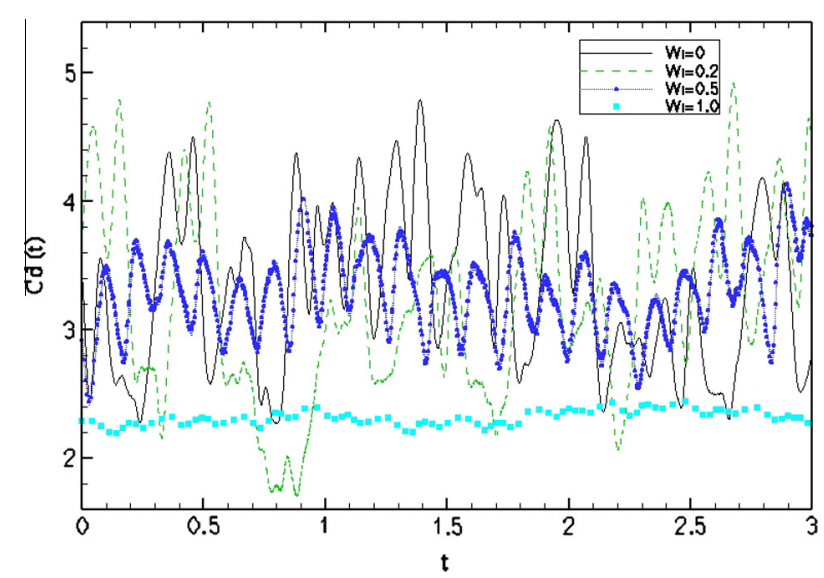

Fig. 10. The evolution of the drag coefficient for $\epsilon=0.01$ and $B=0.1$ at $R e=5000$.

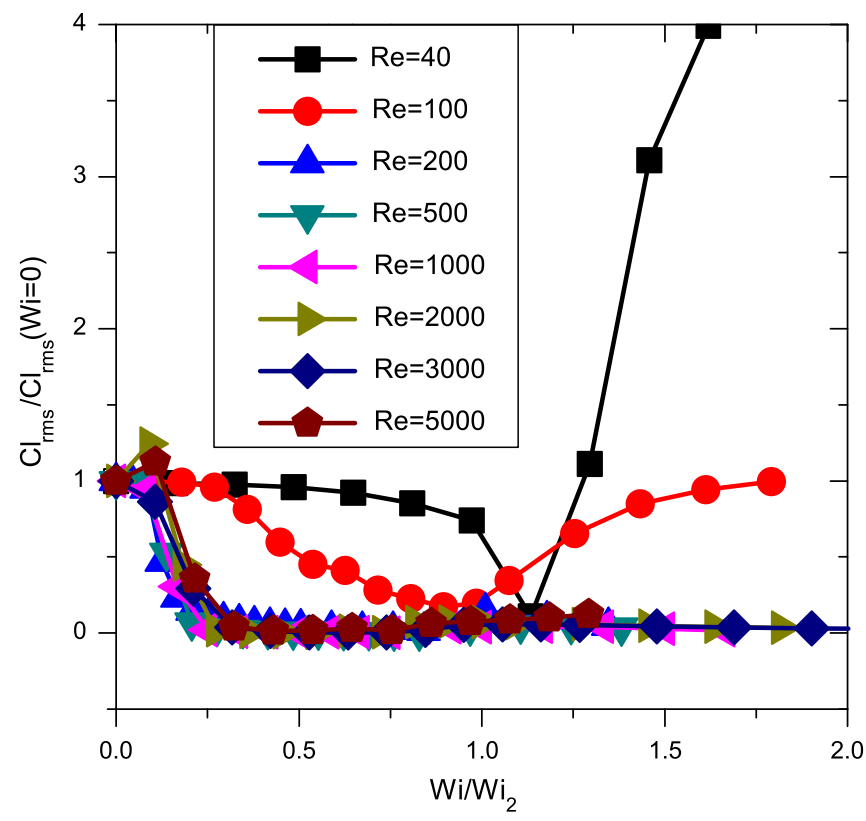

Fig. 11. Root mean square lift force reduction by the polymer additive for $\epsilon=0.01$ and $B=0.1$.

$\epsilon$. Despite the fact that the flow recovers the Newtonian flow at $\epsilon=0$, the results at small values of $\epsilon$ that may show a transition to the Newtonian limit, as expected theoretically for high Reynolds number, are difficult to obtain as numerical instabilities arise. At high Reynolds number, reducing $\epsilon$ in order to make the flow ap proach the Newtonian flow, causes the turbulent fluctuations to in crease making for very high local Weissenberg numbers which leads to the break down of the calculations. Here our selection of $\epsilon$ values does show an change of the drag but only zone for low Weissenberg numbers in Fig. 5b or intermediate Reynolds num bers for $W i=2.0$ in Fig. 6 . For higher values the drag has already saturated most probably due to strong non linear effects. Our Figs. $5 \mathrm{~b}$ and 6 seem however to be consistent with observations of turbulent drag reduction by polymer additives since the re quired concentrations of polymer are very low (parts per million by weight) and further increase of polymer concentration makes no contribution to drag reduction [31]. We therefore use $\epsilon=0.01$ for the rest of the calculations in this paper.

\section{Drag enhancement and drag reduction}

Both drag enhancement and drag reduction have been observed in our numerical results [53]. A phase diagram in Fig. 7a shows the drag behavior in the parameter space $R e$ Wi. The behavior of the drag can be identified by the two threshold Weissenberg numbers $\left(W i_{1}(R e)\right.$ and $\left.W i_{2}(R e)\right)$ as a function of Reynolds number. The threshold Weissenberg numbers are pointed out in Fig. 7b. Below a first threshold value noted $W i_{1}$ the drag behaves like for the New tonian fluid. Between $W i_{1}$ and a second threshold value $W i_{2}$, the drag of the cylinder is reduced. Above $W i_{2}$, only drag enhancement is observed. The values obtained for $W i_{2}$ as a function of $R e$ are listed in Table 6. As the Reynolds number increases, the drag behavior of the cylinder transits from two regimes to three re gimes. This transition occurs near a Reynolds number close to $R e=40$, which coincides with the vortex shedding regime. This points out the strong dependence of the drag behavior on the structure of the flow. This diagram which spans roughly 5 decades in Reynolds number summarizes our findings on drag behavior of the cylinder and shows that both drag enhancement and drag reduction can be obtained but only for specific parts of the param eter space. The drag reduction zone narrows slightly in the phase diagram above the transition and then keeps roughly the same span in Wi as the Reynolds number increases. For $R e \leqslant 40$, there is no vortex shedding for the Newtonian case. The drag, however, shows enhancement for the non Newtonian case. The drag in creases as the Weissenberg number increases above $W i_{2}$. For $R e>40$, the wake of the cylinder becomes unstable for the Newto nian fluid; for the non Newtonian case drag reduction is achieved between two threshold Weissenberg numbers. The drag reduction goes through a maximum at the center of this region. The maxi mum drag reduction turns out to be near $50 \%$ and is practically independent of the Reynolds number in the asymptotic large Rey nolds number limit. Above $\mathrm{Wi}_{2}$, The drag enhancement of the cyl inder increases as the Weissenberg number increases and can exceed $250 \%$ in our simulations. Fig. $7 \mathrm{~b}$ displays the drag behavior at two Reynolds numbers ( 10 and 500 ) to give a quantitative illus tration of the effect of the viscoelasticity. We plot the drag of the cylinder (normalized by the Newtonian drag) for different Rey nolds numbers versus the Weissenberg number in Fig. 8. This fig ure clearly shows the difference between the low Reynolds number and the high Reynolds number behaviors. For the low Rey nolds number situation, only drag enhancement is observed above

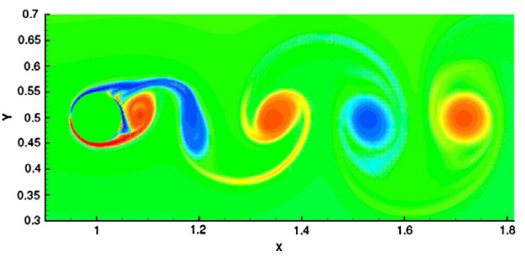

(a) $\mathrm{Wi}=0$

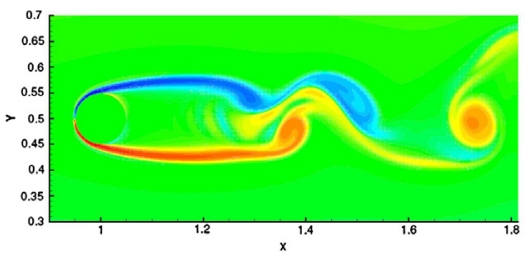

(b) $\mathrm{Wi}=2$

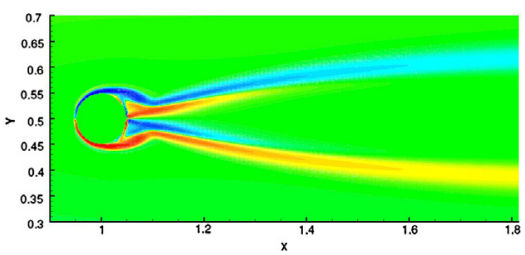

(c) $\mathrm{Wi}=6$

Fig. 12. Vorticity snapshot for $\epsilon=0.01$ and $B=0.1$ at $R e=1000$. 
a value of 1 for the reduced Weissenberg number $\left(W i / W i_{2}\right)$. On the other hand for the high Reynolds numbers, drag reduction is ob served in an interval of reduced Weissenberg numbers below 1

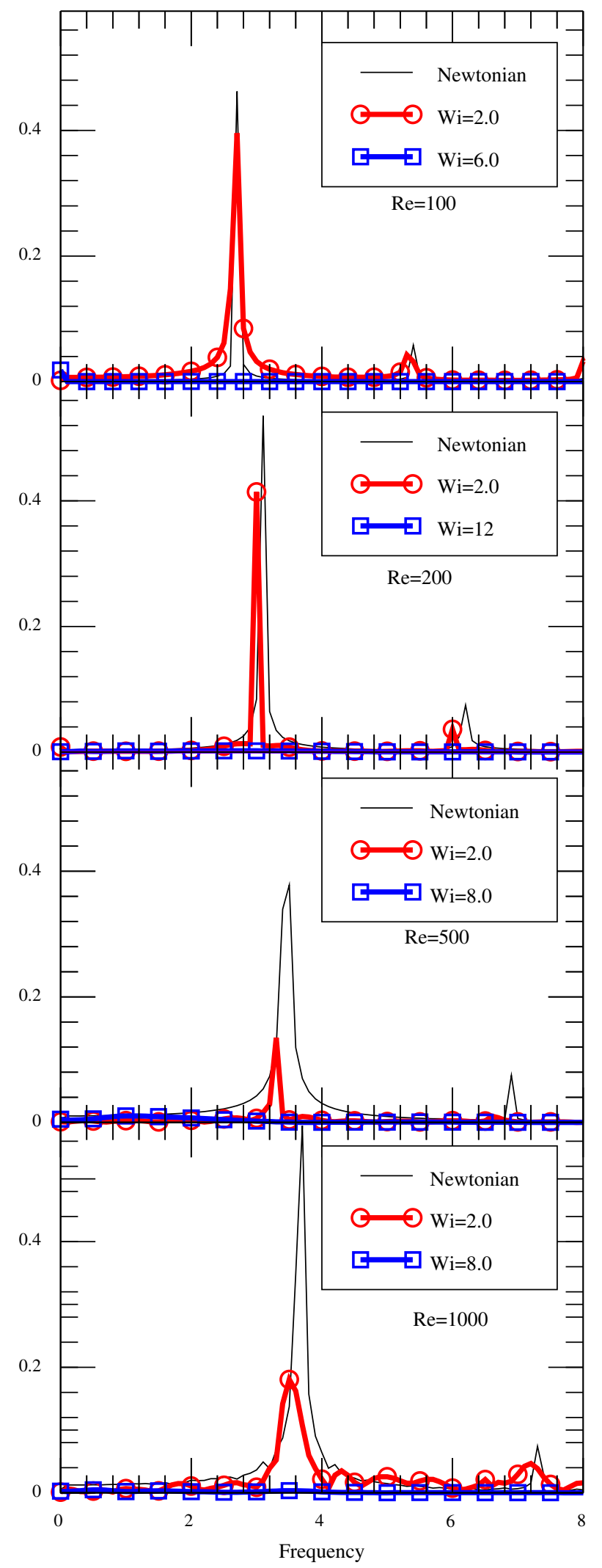

Fig. 13. The power spectrum of transverse-velocity variations downstream of the cylinder. and whose extent increases as the Reynolds number increases. The extent of this range then saturates for even higher $R e$ as shown in Fig. 8. The values of the increase in drag do not collapse perfectly for values of the reduced $W i / W i_{2}$ above 1 . When the reduced Weissenberg number is lower than a value of 1 , the low Reynolds numbers show a plateau at a relative drag of 1 showing Newtonian behavior while on the other hand drag reduction is achieved for higher values of the Reynolds number $(R e>40)$. The maximum drag reduction increases as the Reynolds number increases and seems to reach a saturation value for a Reynolds number near 2000.

When the wake is unsteady, the lift force is also important for the immersed body. The lift and drag forces are unsteady and oscil late. This oscillation of the forces is important and interesting for many engineering problems. Fig. 9 shows the phase portrait of the drag and lift forces. For the two dimensional flow, the vortex shedding is regular when the Reynolds number is less than 1000 , the drag and lift force show a periodic behavior in the phase por trait. From Fig. 9, the amplitudes of both drag and lift forces in crease as the Reynolds number increases for the Newtonian fluid $(W i=0)$. The amplitude of the lift force is larger than the amplitude of the drag force, a low drag force corresponds to a low lift force. For the polymer solutions, the force phase portrait is almost super imposed to the one obtained with the Newtonian fluid when the Weissenberg number is smaller than $W i_{1}$, then both the absolute value of the drag and the amplitude of the drag or lift forces are re duced quickly when the Weissenberg number is larger than $W i_{1}$. In fact, the phase portrait of the drag and lift forces converges to a point before the Weissenberg number exceeds $W i_{2}$.

When the Reynolds number increases, the flow becomes turbu lent, the drag and lift forces are too chaotic to be plotted. Fig. 10 shows the evolution of the drag coefficient versus time. The lift coefficient has a similar evolution. We can observe that the drag shows low frequency oscillations due to the vortex shedding. The amplitude of the drag fluctuations is very large for the Newtonian fluid and for $W i=0.2$. As the Weissenberg number increases, the amplitude of the drag fluctuations decreases $(W i=0.5)$, even if the mean drag force does not change. Whereafter, both the mean value and the fluctuations decrease at $W i=1$, the drag keeps a con stant value with time for higher Weissenberg numbers. This sug gests several steps for the drag behavior for the viscoelastic turbulent flow. The flow is not affected by elasticity at very small Weissenberg numbers. After the Weissenberg number exceeds $W i_{1}$, both the mean drag and the fluctuations continue to decrease. The drag then becomes roughly constant with time.

Fig. 11 shows the root mean square lift force reduction due to the polymer additive. When the Reynolds number is greater than 200 , the root mean square lift force $\left(\mathrm{Cl}_{r m s}\right)$ becomes negligible as soon as the Weissenberg number is greater than $0.25 W i_{2}$. Espe cially, for the Reynolds numbers 40 or 100 , the $C_{r m s}$ first reduces and gets a minimum value nearby $W i_{2}$, then it increases with the Weissenberg number. We have performed these two cases with a very long time simulation, but the lift force keeps a constant value with time at high Weissenberg numbers. It means the flow field is steady but loses up down symmetry, which will be discussed in Section 6.2 .

\section{The flow modification by polymer}

\subsection{The two regimes}

One of the main features of the modifications of the flow by the presence of polymers is shown in Fig. 12. We plot vorticity snap shots for a Reynolds number of 1000 with three different Weiss enberg numbers to display the three different flow regimes. One 
of the most striking observations is the disappearance of vortex shedding behind the cylinder for $W i=6.0$. Another interesting fea ture is the increase in the length of the wake in the drag reduction regime pushing away the pressure well, this is consistent with the two dimensional simulation of [39] and the latest three dimensional simulations [43]. When the shedding length becomes too long, the shedding is no longer observed due to the limitation in the channel length used. At this stage, drag of the cylinder is en hanced dramatically. These two features in the dilute polymer solutions correspond to the two regimes of the cylinder drag: drag enhancement and drag reduction. In the drag reduction regime, the distance of two adjoining vortices is longer than that of the New tonian fluid. This feature can be appreciated from the discrete Fou rier transforms of transverse velocity variations downstream of the cylinder as shown in Fig. 13. The frequency of the peak increases as the Reynolds number increases for the Newtonian fluid. However the peak, which corresponds to the vortex shedding frequency, moves toward the low frequency zone as the Weissenberg number increases. This suggests the decrease of the Strouhal number with polymer additives for the intermediate Reynolds numbers, which is consistent with the two dimensional simulations [39] and experimental observations carried out using soap films as quasi two dimensional fluids [13]. We also note that the height of this peak decreases as the Weissenberg number increases until it disap pears altogether in the drag enhancement regime for all the Rey nolds numbers examined. Furthermore, for the drag reduction regime and for the high Reynolds numbers, it seems that the amplitude of the higher harmonics increases with respect to the Newtonian case indicating a large deformation of the wake.

As we have seen earlier, the drag behavior shows a strong coupling with the flow behavior. The nontrivial drag reduction happens only for the unstable regime (i.e. $R e \geqslant 40$ ), drag enhancement follows along with the inhibition of the shedding. We illustrate the effect of polymer and the different drag regimes using $R e=10$ and $R e=100$. Fig. 14 gives the streamwise velocity profiles at five different positions. The differences between the Newtonian fluid and the viscoelastic fluids are clear. At $x=1$ that is the position of the center of the cylinder, the velocity profiles are very different from Poiseuille flow, the maximum velocity oc curs very close to the cylinder. Downstream of the cylinder at $x=2$, the streamwise velocity has a local minimum near the cen terline of the channel for every profile. As the Weissenberg number increases, the local minimum velocity decreases. Farther down stream of the cylinder at $x=3$ and $x=4$, the flow is on the way to recover the Poiseuille flow, and the differences in profiles be comes even less obvious at $R e=10$. When the Weissenberg num ber is less than $W i_{1}$, the streamwise velocity profiles are the same than for the Newtonian fluid. The polymer changes dramat ically the flow for $W i>W i_{1}$. The most obvious effect occurs at the wake of the cylinder at $R e=100$, the streamwise velocity at high Weissenberg number is slower to recover the undisturbed bulk velocity than the Newtonian fluid. In this case there is no more shedding and a drag enhancement is obtained as shown in Fig. 7.

In order to further examine the behavior of viscoelastic flows around the cylinder, we plot in Figs. 15 and 16, the pressure vari ation, the streamwise velocity, the elongation rate as well as the longitudinal elastic stress along the centerline of the channel. For $R e=10$ in Fig. 15, the streamwise velocity recovers more quickly than the Newtonian fluid at the rear of the cylinder $\left(\left(\begin{array}{ll}x & x_{c}\end{array}\right) / R\right.$ higher but close to 1$)$ specially for high Wi. But since the pressure also increases in this region, the pressure is not sufficient to ex plain the higher velocity for the non Newtonian case. Therefore we plotted $\sigma_{x x}$ in Fig. 16 to find the driving force for the accelera tion of local fluid particles. The plot of $\sigma_{x x}$ shows a large increase

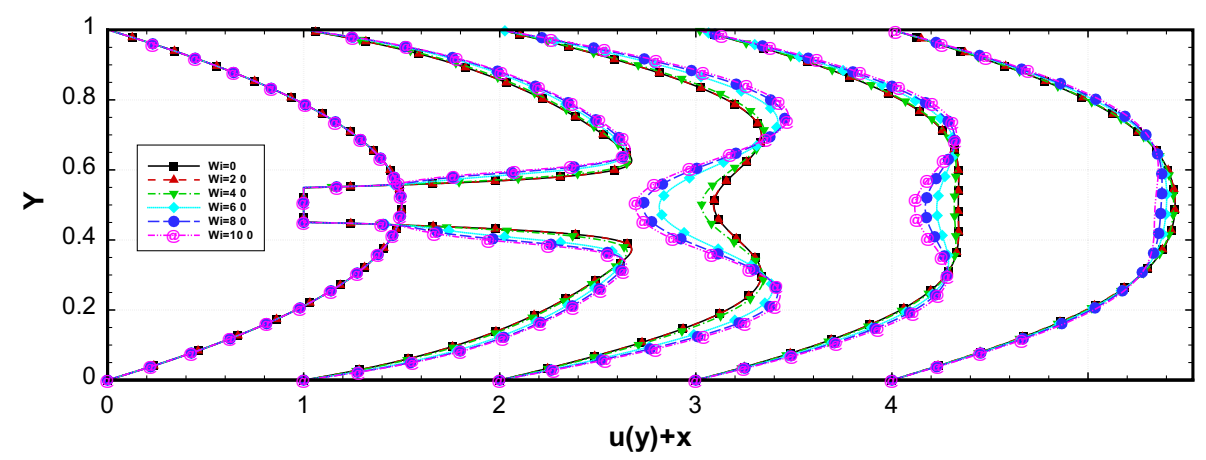

(a) $R e=10$

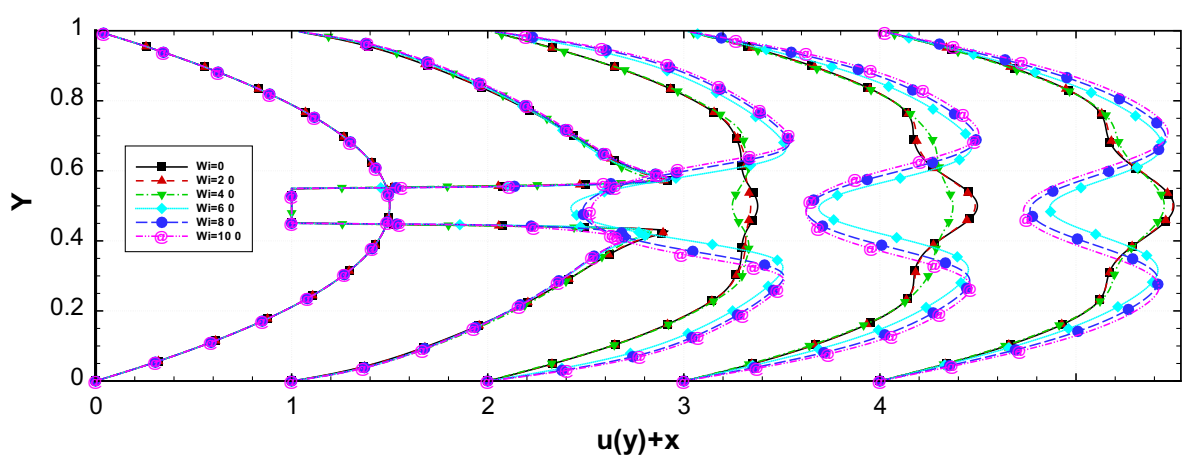

(b) $R e=100$

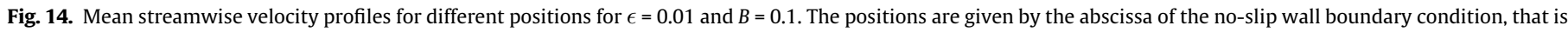
$x=0,1,2,3$ and 4 . 
over a very short distance near the cylinder culminating at a very high maximal value near the rear of the cylinder. This sharp in crease of the longitudinal elastic stress will produce a very strong driving force to increase the pressure and accelerate the local fluid particles. After the peak, $\sigma_{x x}$ decreases as the distance to the cylin der increases, this will decelerate the local fluid. The streamwise velocity will therefore increase more slowly than the Newtonian fluid as observed. For $R e=100$, there exists a recirculation bubble behind the cylinder even though the wake becomes unsteady.
Downstream of the cylinder, the base pressure becomes higher for $W i \geqslant 4.0$ as compared to the Newtonian base pressure. The length of the mean recirculation bubble increases. The velocity profiles at high Weissenberg number show the elongation of the recirculation bubble for the mean flow. In the drag enhancement regime, however, the base pressure decreases and the mean recir culation bubble disappears.

Note that for $W i>W i_{1}$, the pressure difference between the up stream and the downstream sides of the cylinder increases as Wi
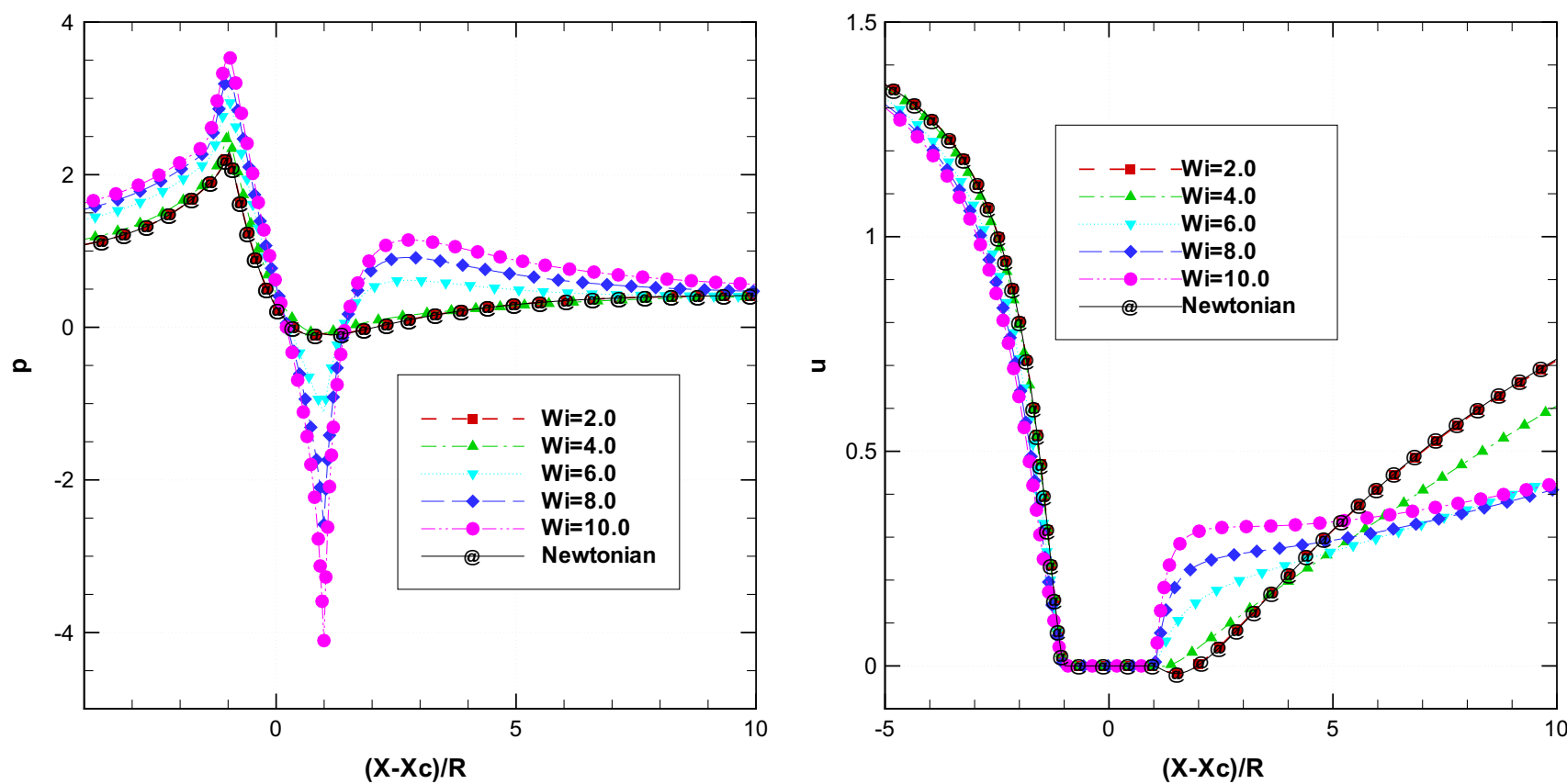

(a) $R e=10$
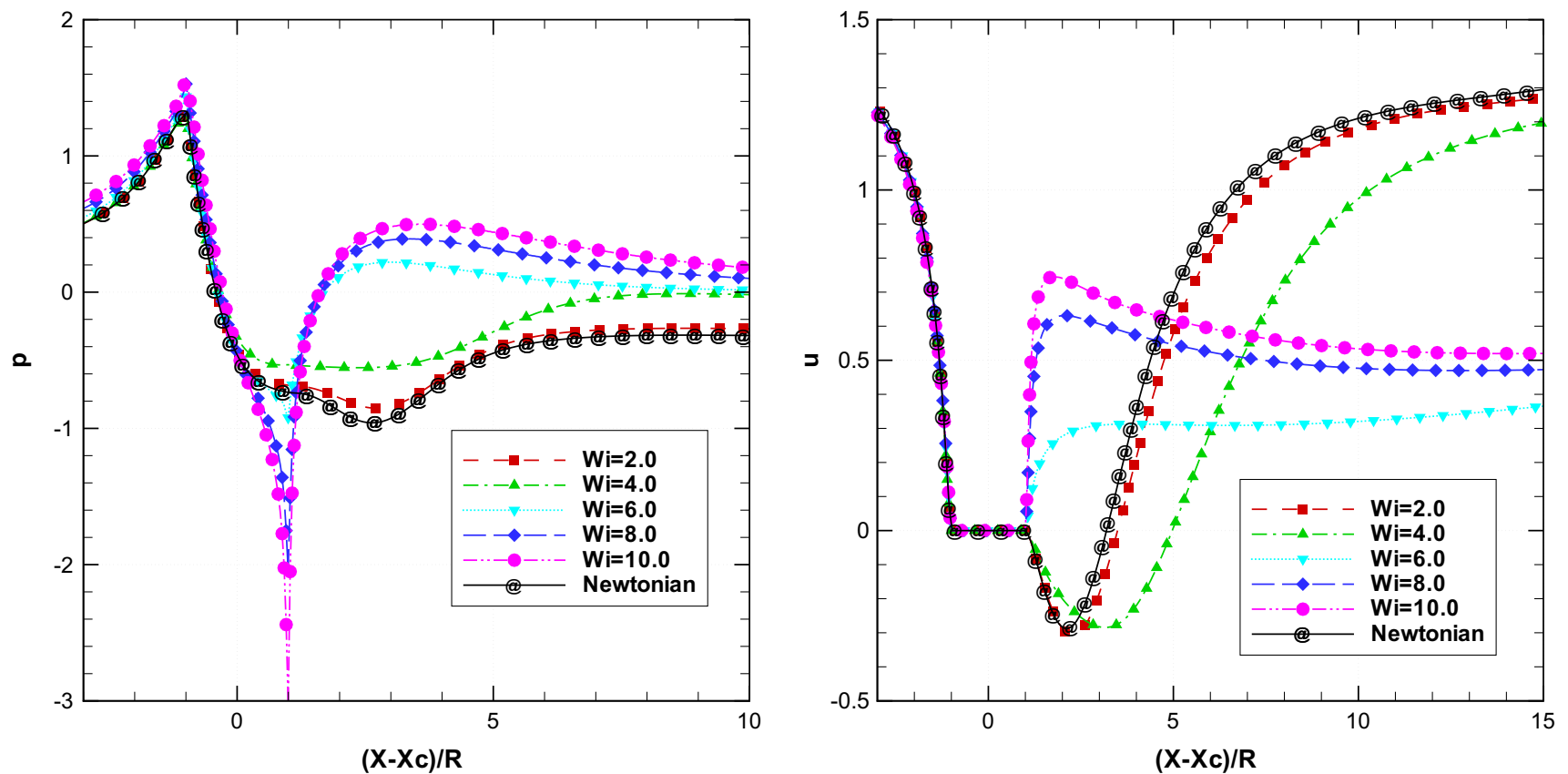

(b) $R e=100$

Fig. 15. Mean non-dimensional pressure and streamwise velocity profiles on the centerline of the channel $(y=0.5)$ for various Weissenberg numbers with $\epsilon=0.01$ and $B=0.1$. 
increases for $R e=10$, i.e. in the drag enhancement regime. How ever, for $R e=100$, the pressure difference is less than for the New tonian flow for Weissenberg numbers between $W i_{1}$ and $W i_{2}$, i.e. in the drag reduction regime. For Weissenberg numbers larger than $W i_{2}$, the pressure at the rear of the cylinder decreases to a much lower value with an obvious peak; the decrease of the pressure at the rear of the cylinder increases in amplitude indicating drag enhancement as the Weissenberg number increases. The two cases therefore illustrate the differences in the variation of the pressure between the drag reduction and the drag enhancement regimes.

Another important feature can be appreciated using the elonga tion rate and its relation to the longitudinal elastic stress along the centerline of the channel. This elongation rate also peaks near the rear of the cylinder as shown in Fig. 16 for high Weissenberg num ber. Large elongations are associated with large elastic stresses as has been observed in recent experiments where large elongations of the polymers was reported in three dimensional experiments in microchannels of flow past a cylinder [16]. Furthermore, the location of the maximum longitudinal stress, and of the elongation, move towards the cylinder wall for the drag enhanced regime (i.e. $W i>W i_{2}$ ), while for the drag reduction regime this maximum moves away from the cylinder.
Let us see the flow behavior when Wi increases. As the Reynolds number increases in a Newtonian fluid, the inertial force increases, causing shear rate enhancements in the shear layer downstream of the wake. The periodic wake behind the cylinder will eventually become turbulent with the vortices spreading into the whole chan nel as the vorticiy snapshot for $R e=5000$ shows in Fig. 17a. In the presence of the viscoelastic fluid however, the irregular wake be comes more ordered as the Weissenberg number increases. When the Weissenberg number increases to $W i=2$, the wake becomes regular, the vortices don't spread everywhere even if the vortices have strong interactions. As the Weissenberg number increases in the drag reduction regime, the vortices shed from the cylinder and show an increased orderly distribution in the channel. Also, the length of the wake increases as the Weissenberg number in creases in the drag reduction regime.

For $W i$ larger than $W i_{2}$, ie. in the drag enhancement regime, the vortices disappear altogether, and the wake becomes a simple two sided shear layer. At even higher $W i$, these shear layers become unstable as in a Kelvin Helmholtz type instability, which is most probably due to increased elastic stresses. We therefore plot the two elements of the conformation tensor $\sigma_{x x}$ and $\sigma_{y y}$ in Fig. 18 Both of them increase as the Weissenberg number increases. In
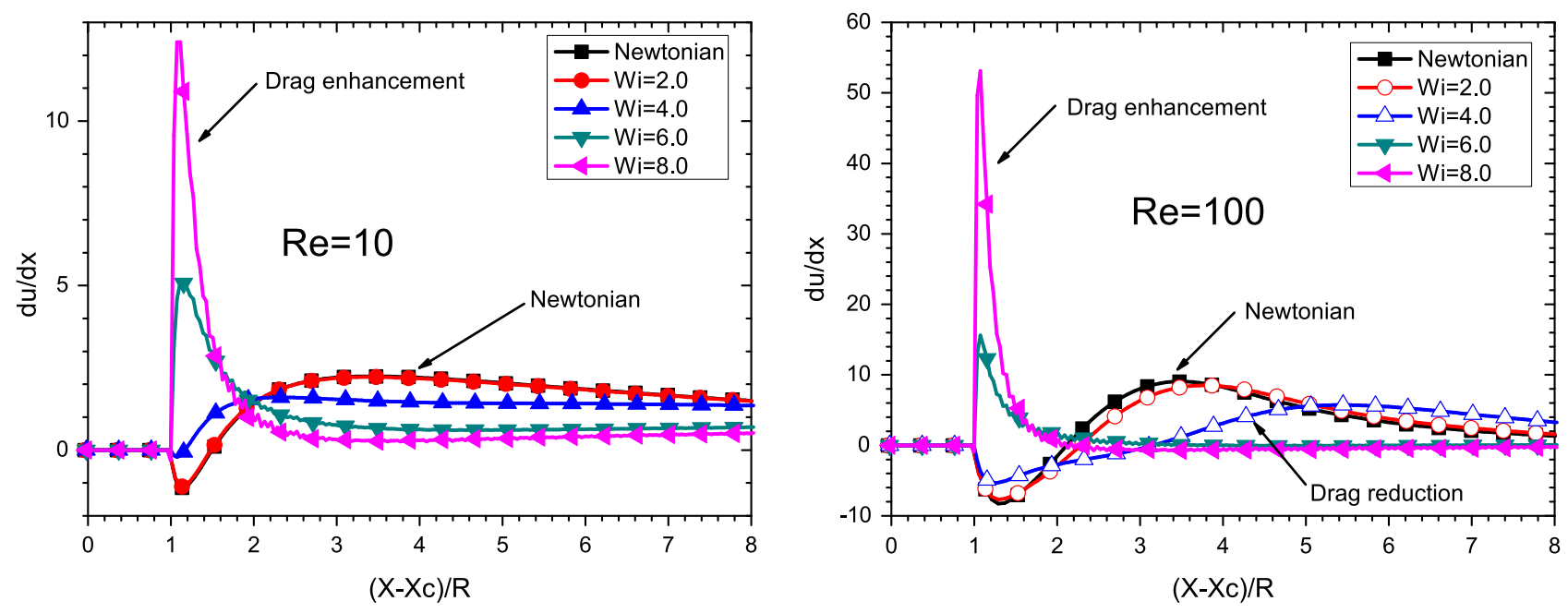

(a) elongation
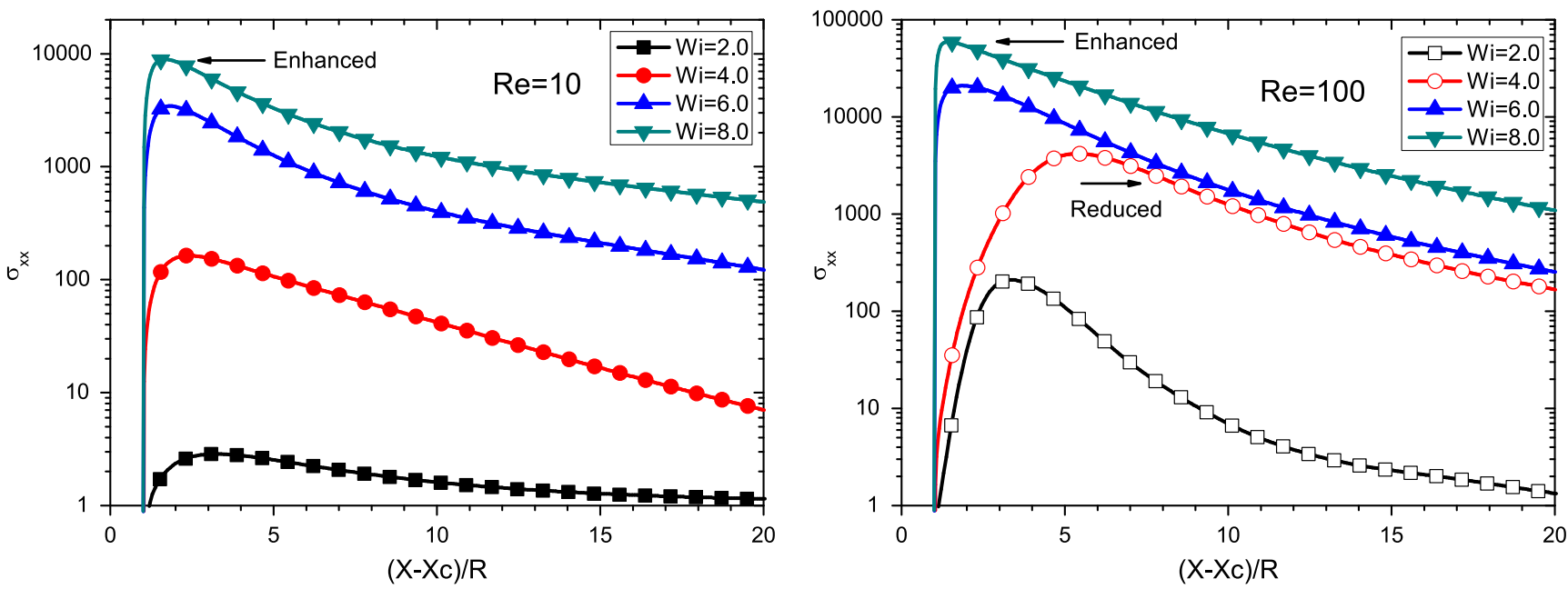

(b) $\sigma_{x x}$

Fig. 16. The elongation and $\sigma_{x x}$ along the centerline of the channel for two different $\operatorname{Re}$ and different $W i$ with $\epsilon=0.01$ and $B=0.1$. 
the contractive zone ahead of the cylinder, $\sigma_{x x}$ is less affected than $\sigma_{y y}$. Both of them are extremely large at the rear of the cylinder. Especially $\sigma_{x x}$ is more affected by the elongational flow. Along the longitudinal direction, both of them decrease by releasing elas tic energy while the instabilities are observed there. The wake characteristics shown here for a single cylinder in a polymer solu tion are also observed in numerical simulations of two dimen sional grid turbulence, the grid being composed of an array of cylinders [54].

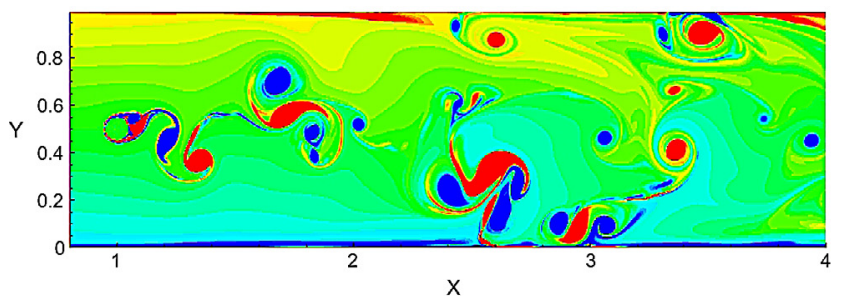

(a) $\mathrm{Wi}=0$

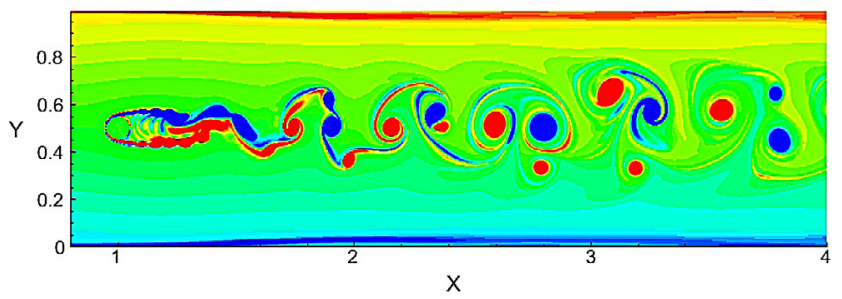

(c) $\mathrm{Wi}=2$

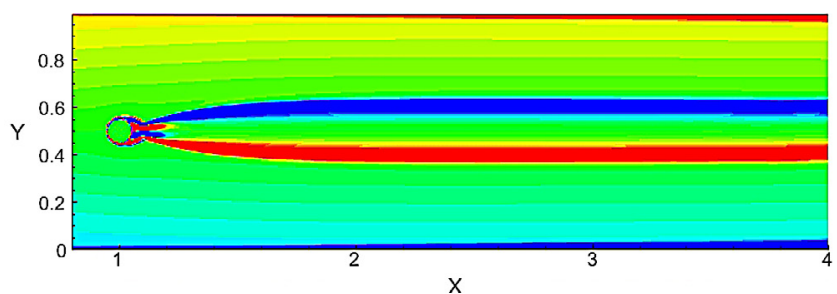

(e) $\mathrm{Wi}=4$
Besides using the instantaneous flow to illustrate the effect of polymers, we show in Fig. 19 the mean streamwise velocity pro files for different Weissenberg numbers. The most obvious feature may be a local lower streamwise velocity downstream of the cylin der for the two Reynolds numbers used and for a Weissenberg number larger than $W i_{2}$. At the same time, a large shear rate occurs for the shear layer resulting from the interaction of the flow with the cylinder. This brings forth the question of how the polymers af fect the elongational rate. Fig. 20 shows the probability density

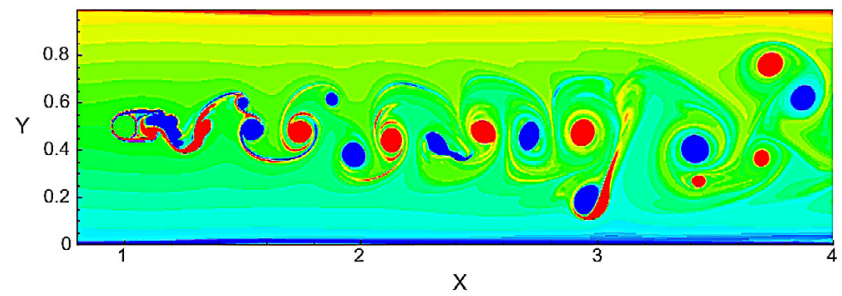

(b) $\mathrm{Wi}=1$

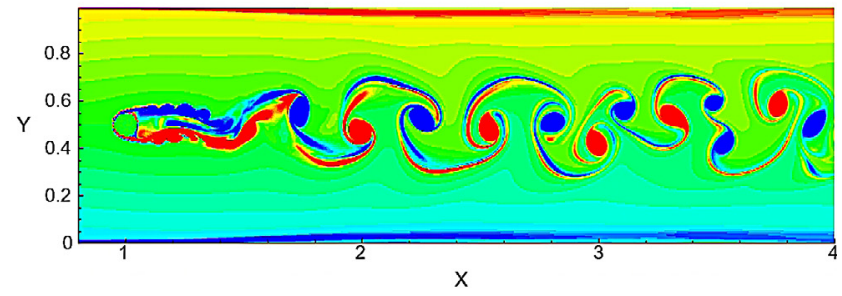

(d) $\mathrm{Wi}=3$

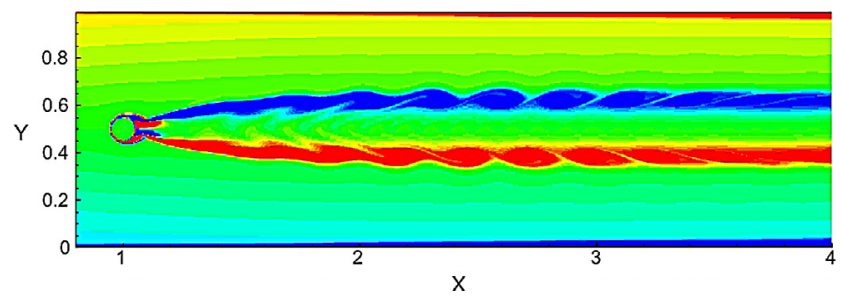

(f) $\mathrm{Wi}=5$

Fig. 17. Vorticity snapshots for $\epsilon=0.01$ and $B=0.1$ at $R e=5000$.

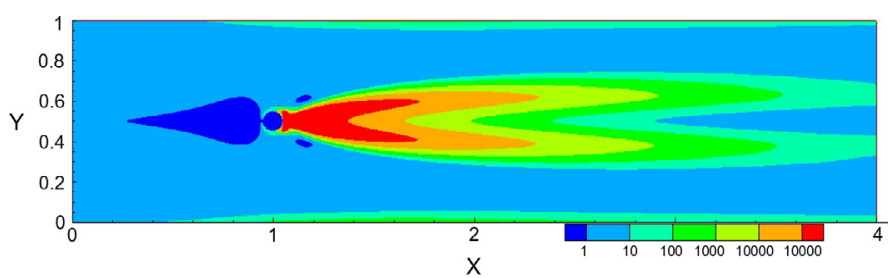

(a) $\sigma_{x x}$ at $\mathrm{Wi}=4$

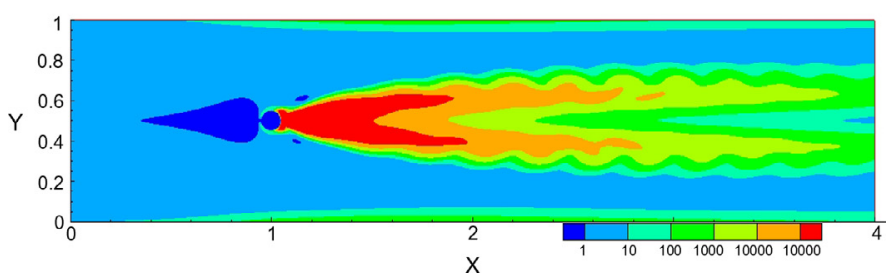

(c) $\sigma_{x x}$ at $\mathrm{Wi}=5$

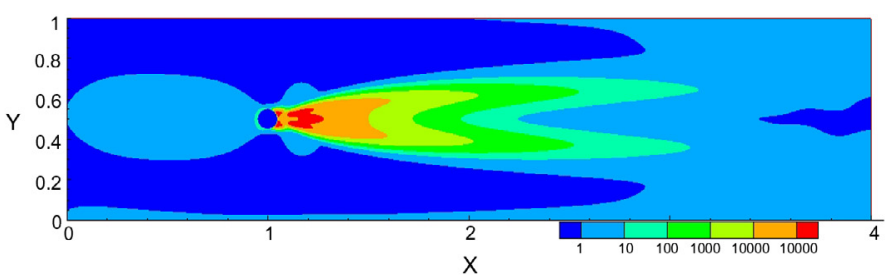

(b) $\sigma_{y y}$ at $\mathrm{Wi}=4$

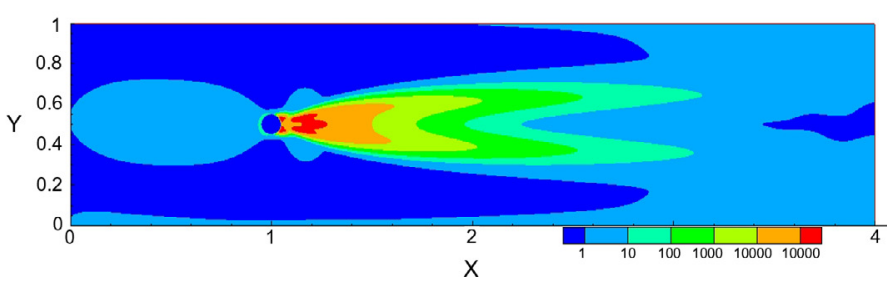

(d) $\sigma_{y y}$ at $\mathrm{Wi}=5$

Fig. 18. $\sigma_{x x}$ and $\sigma_{y y}$ snapshots for $\epsilon=0.01$ and $B=0.1$ at $R e=5000$. 
function of $d u / d x$ in a small square window and for a long statistic period. The window used is located between 2.5 and 3 in the streamwise direction and between 0.25 and 0.75 in the transverse direction. The width of the probability density function of the gra dient of longitudinal velocity shrinks as the Weissenberg number increases as seen in this Fig. 20, indicating a suppression of elonga tional rate by the increasing fluid elasticity. Thus, while the poly mer seems to have increased the shear rate, very large elongational rates seem to have been suppressed in the wake re gion as it has been observed in recent experiments [25].

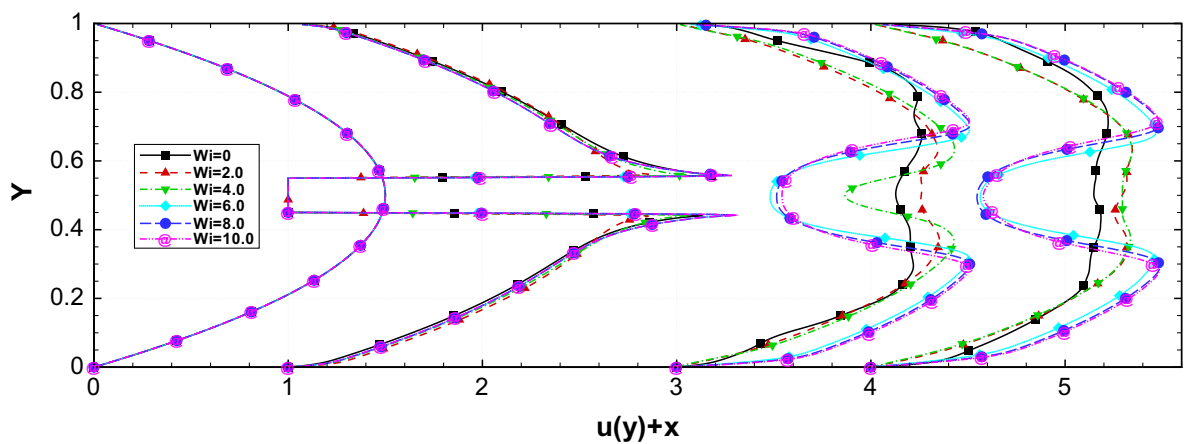

(a) $R e=3000$

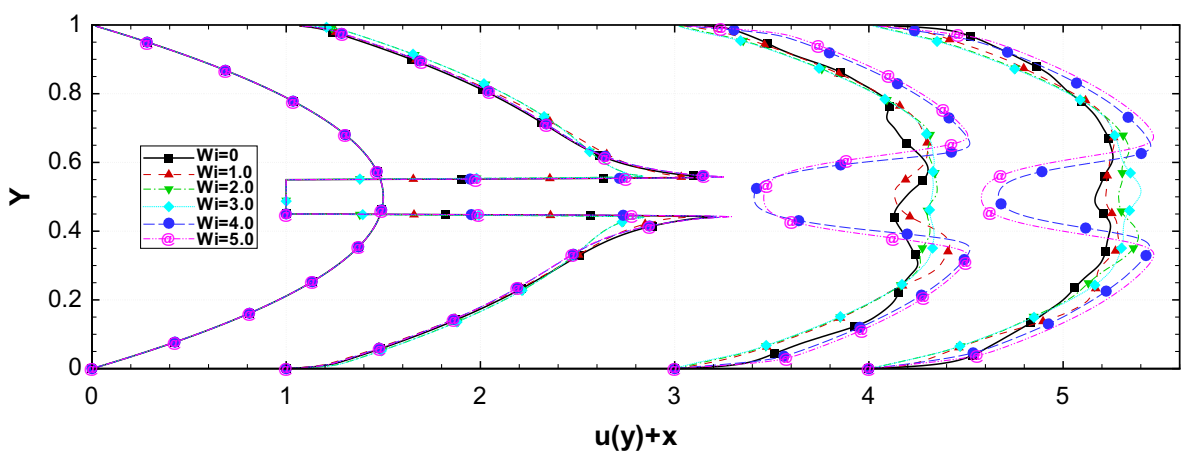

(b) $R e=5000$

Fig. 19. Mean streamwise velocity profiles for different positions for $\epsilon=0.01$ and $B=0.1$.

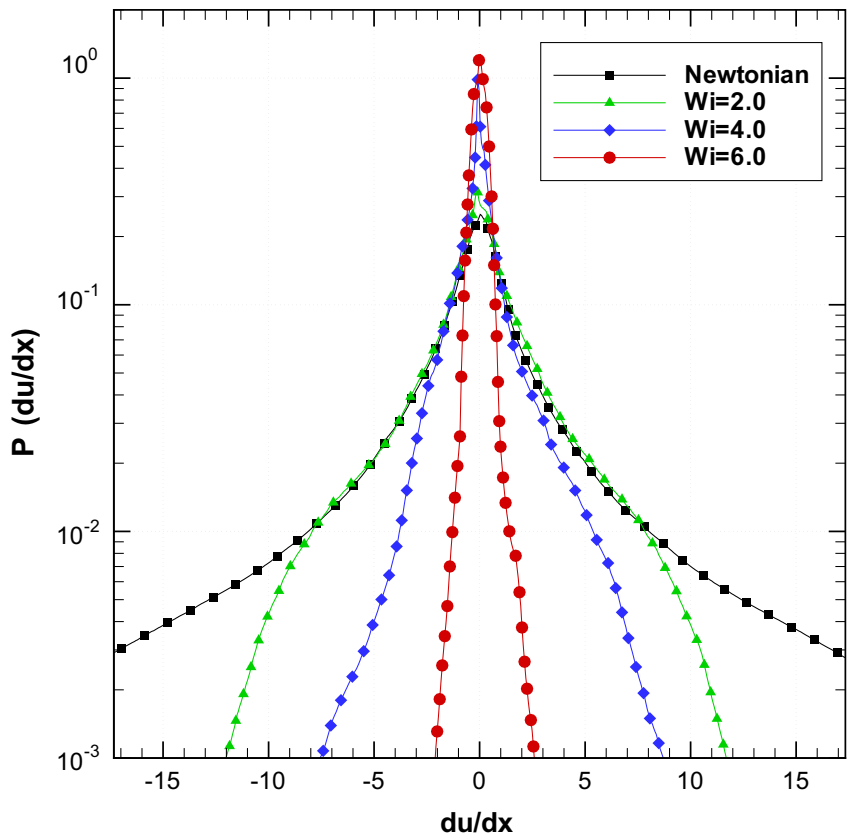

(a) $R e=3000$

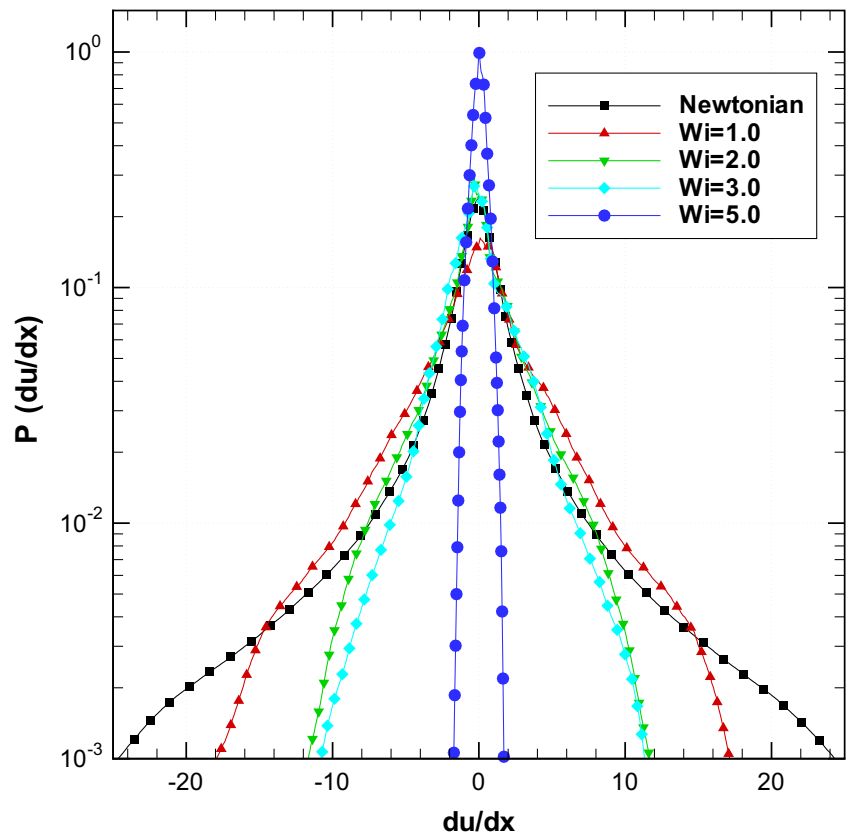

(b) $R e=5000$

Fig. 20. Probability density function of the elongation rate in the square window in wake for $\epsilon=0.01$ and $B=0.1$. 


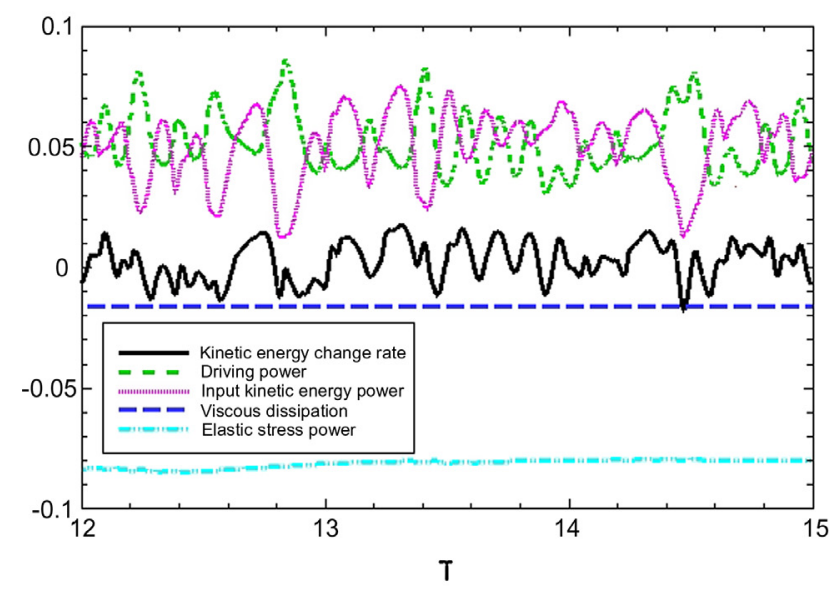

(a) The total energy balance at $W i=3$

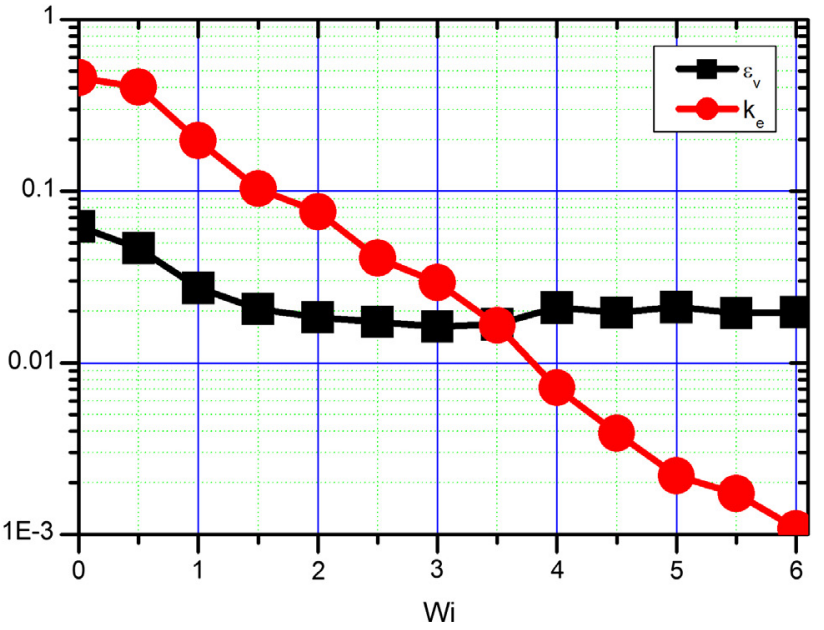

(b) $k_{e}$ and $\epsilon_{v}$

Fig. 21. The total energy of the domain for $\epsilon=0.01$ and $B=0.1$ at $R e=5000$.

Table 7

Summary of the principal global quantities for $\epsilon=0.01$ and $B=0.1$ at $\operatorname{Re}=5000$.

\begin{tabular}{llllll}
\hline$W i$ & $\left\langle\epsilon_{v}\right\rangle$ & $\left\langle\epsilon_{p}\right\rangle$ & $\left\langle k_{e}\right\rangle$ & $\left\langle E_{p}\right\rangle$ & $\left\langle P_{f}\right\rangle$ \\
\hline 0 & 0.0622 & 0 & 0.4585 & 0 & 0.062 \\
1 & 0.0274 & 0.0419 & 0.1973 & 0.0021 & 0.070 \\
2 & 0.0184 & 0.0691 & 0.0759 & 0.0069 & 0.087 \\
3 & 0.0162 & 0.0866 & 0.0293 & 0.0130 & 0.103 \\
4 & 0.0210 & 0.1310 & 0.0072 & 0.0257 & 0.155 \\
5 & 0.0211 & 0.1789 & 0.0022 & 0.0409 & 0.179 \\
\hline
\end{tabular}

At high Reynolds numbers, several investigations show that the polymer suppresses the vortices at different scales $[25,36,54]$ for two dimensional turbulence, but how the polymer affects the glo bal quantities for a single cylinder in the flow is still unclear. We therefore checked the energy balance for the present cases. From the momentum equation and the constitutive equation in the gov erning equations written above, the kinetic and elastic energy are given by $\left\langle\frac{D E_{k}}{D t}\right\rangle \quad\left\langle\epsilon_{v}\right\rangle \quad\left\langle\epsilon_{p}\right\rangle+\left\langle P_{f}\right\rangle$ and $\left\langle\frac{D E_{p}}{D t}\right\rangle \quad\left\langle\epsilon_{p}\right\rangle \quad\left\langle\frac{E_{p}}{\tau}\right\rangle$, wherein \langle\rangle$\quad \int d V$ and $V$ is the total volume of the computational domain.

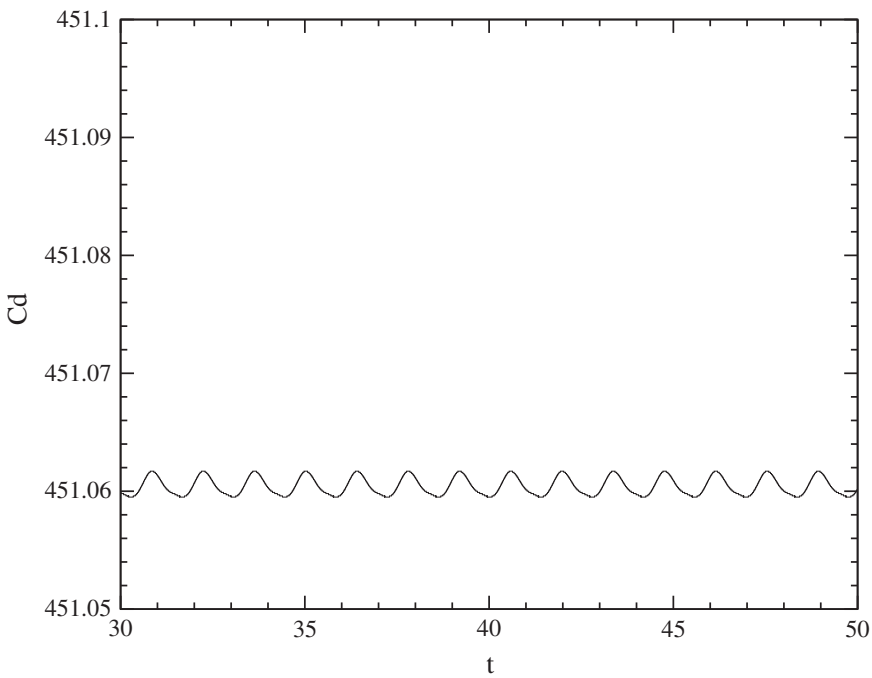

(a) $W i=10$
These two equations indicate the energy transfer relation between polymer and flow of solvent. For our cases, a statistic steadiness yields that the left hand side is zero. The sum of the two equations gives the energy balance relation. The forcing power $\left\langle P_{f}\right\rangle$ is con sumed by the viscous dissipation and the relaxation of the elastic energy of the polymer. The forcing power is mainly composed of the driving work due to the pressure difference between the inlet and outlet boundaries. The rate of viscous dissipation $\left\langle\epsilon_{\nu}\right\rangle=\left(\begin{array}{ll}1 & \epsilon\end{array}\right)$ $\left\langle|w|^{2}\right\rangle /$ Re can be obtained from the vorticity of the fluid, and the elastic stress power $\left\langle\epsilon_{p}\right\rangle \quad \frac{\epsilon}{R e \cdot W i}\left\langle\sigma_{x x} \frac{\partial u}{\partial x}+\sigma_{x y}\left(\frac{\partial u}{\partial y}+\frac{\partial v}{\partial x}\right)+\sigma_{y y} \frac{\partial v}{\partial y}\right\rangle$ extracts kinetic energy from the flow and transfers it into polymer stretching. The elastic energy [34] of Oldroyd B model is given by $\left\langle E_{p}\right\rangle \frac{1}{2} \frac{\epsilon}{R e \cdot W i}$ $\left\langle\sigma_{x x}+\sigma_{y y} \quad 2\right\rangle$.

A typical kinetic energy balance is plotted in Fig. 21a for $W i=3$. The rate of change of the kinetic energy has a zero sum: the input energy is balanced by dissipation due to the fluid viscosity and elasticity. Our results show that the balance between the different terms is well achieved. The average kinetic energy due to the velocity fluctuations, $k_{e}=0.5\left(u^{\prime 2}+v^{2}\right)$, and the viscous dissipation are plotted in Fig. 21b versus the Weissenberg number for

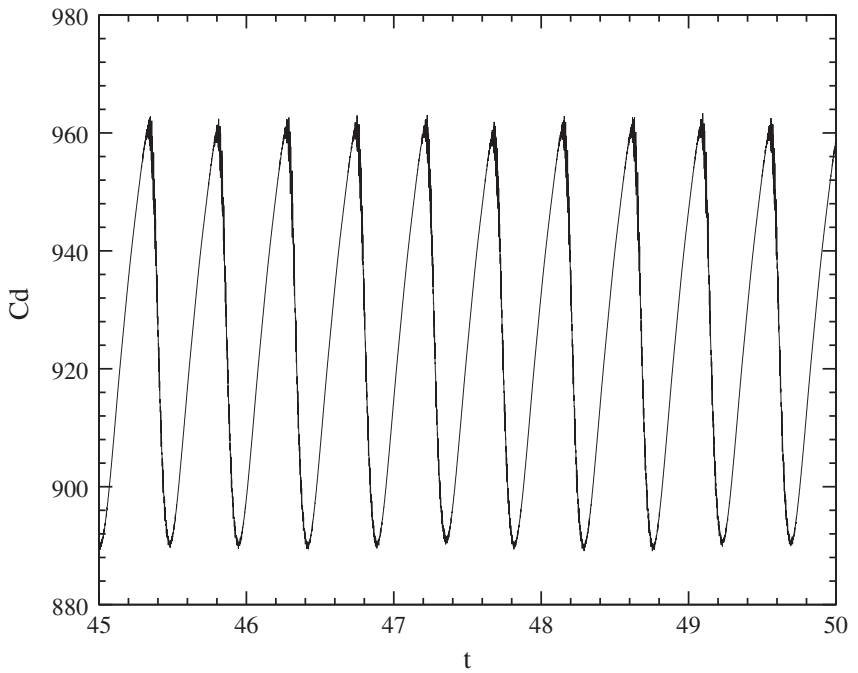

(b) $W i=24$

Fig. 22. The evolution of the drag coefficient on the cylinder for $\epsilon=0.04$ and $B=0.1$ at $R e=0.1$. 


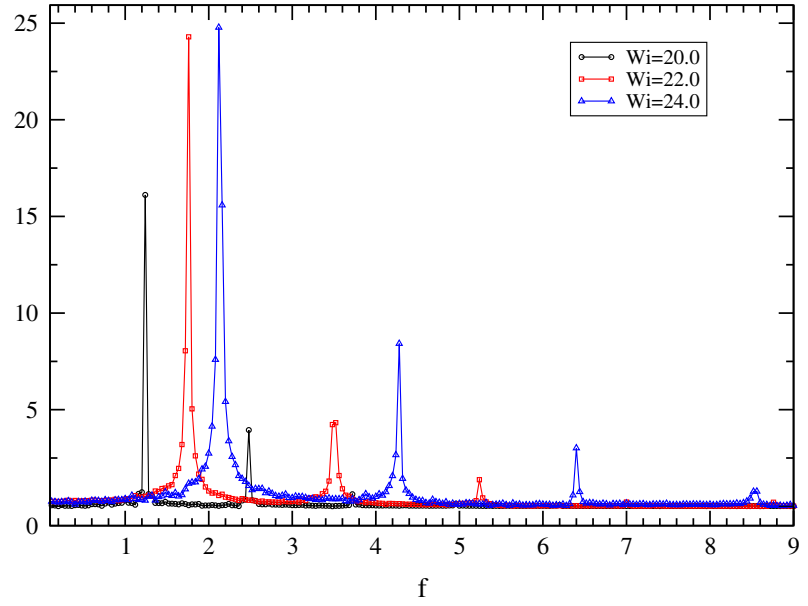

(a) Spectrum of the drag coefficient

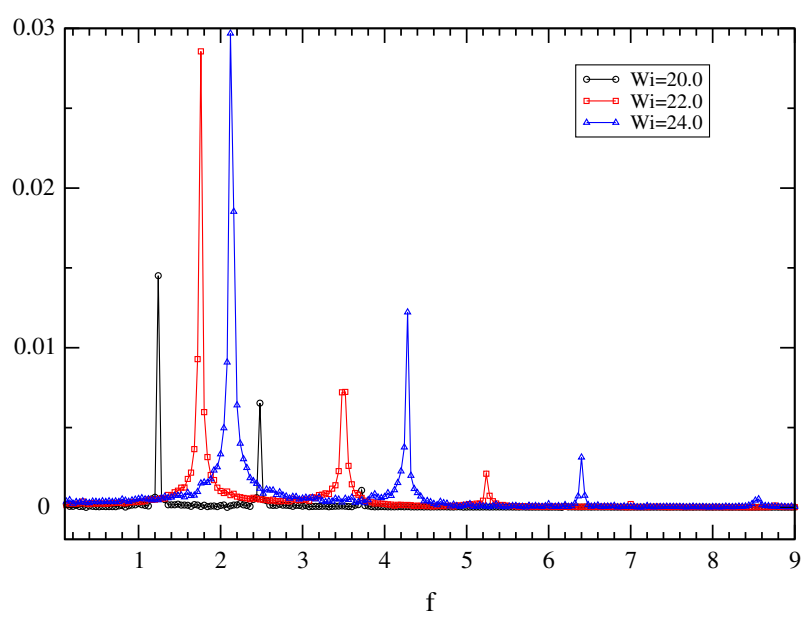

(b) Spectrum of the streamwise velocity

Fig. 23. The discrete Fourier transform (DFT) of the drag and of the streamwise velocity at monitoring point $\left(X_{c}+3 D, Y_{c}-D\right)$ for $\epsilon=0.01, B=0.1$ and $R e=0.1$.

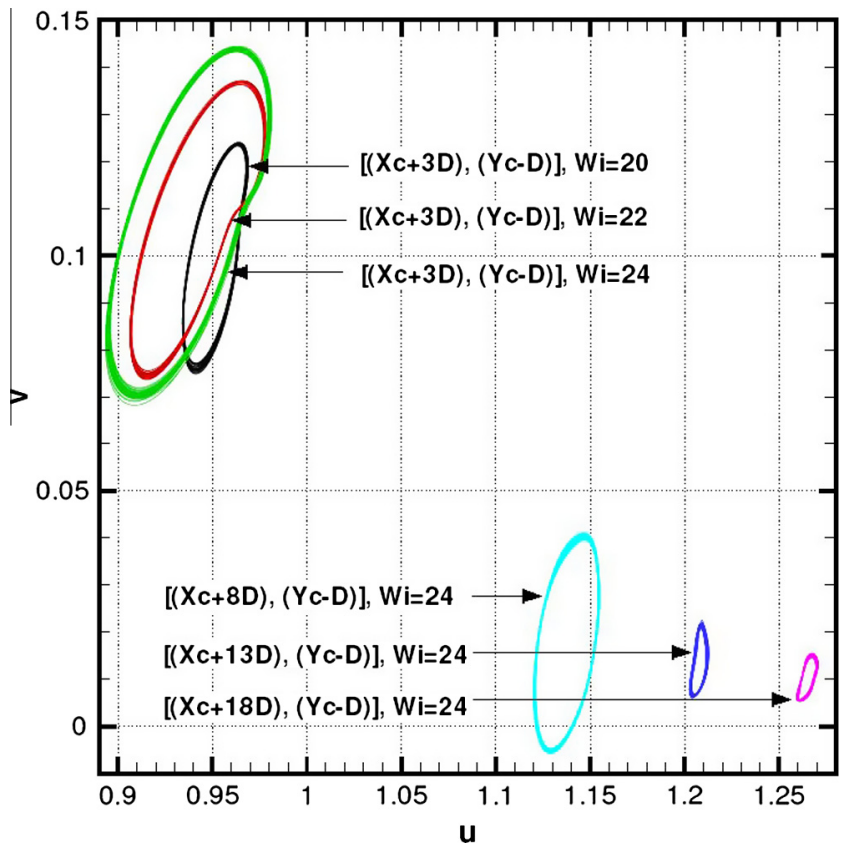

Fig. 24. The phase portrait at four monitoring points for $\epsilon=0.01, B=0.1$ and $\operatorname{Re}=0.1$.

$R e=5000$. The figure shows that the kinetic energy decreases as the Weissenberg number increases in the drag reduction and drag enhancement regimes. However the viscous dissipation has not the similar monotonous behavior, it decreases firstly and then in creases as the Weissenberg number increases. Therefore the mini mum viscous dissipation rather than kinetic energy in the fluid field corresponds to the Weissenberg number with the minimum drag. More physical quantities such as the elastic energy, elastic stress power and the forcing power are listed in Table 7. They are all increasing as the Weissenberg number increases. The kinetic energy decreases monotonously as the Weissenberg number in creases showing that the increase of elastic effects inhibits the fluc tuations in the turbulent wake.

\subsection{Results and discussion on high Weissenberg number instability}

As the Weissenberg number increases, the elastic effects in the fluid increase. This may result in a pure elastic instability or even elastic turbulence at vanishingly small Reynolds numbers [17] The wake instability in viscoelastic flow past confined circular cyl inders was reported in recent experiments [33] and finite volume simulations [38] at small Reynolds numbers. Oliveira and Miranda simulated the flow past a cylinder placed symmetrically in a chan nel, with a blockage ratio of 0.5 , with a FENE CR constitutive mod el. They observed a flow transition from steady to unsteady at a critical Weissenberg number of 1.3 when the solvent to the poly mer solution viscosity ratio is 0.59 . In our results, we found similar unsteady drag for $W i=10$ in dilute polymer solution at $R e=0.1$ with a blockage ratio 0.1 for $\epsilon=0.04$ (see Fig. 22). The amplitude of the drag is very low at $W i=10$, but quite important at $W i=24$. As we see in the figure, the amplitude of the drag oscillations is about $6 \%$ of the mean drag at $W i=24$. Furthermore, the frequency of the drag oscillation also increases. Fig. 23 shows the discrete Fourier transform of the drag and of the velocity at the monitoring point $\left(X_{c}+3 D, Y_{c} \quad D\right)$. Both the drag and the streamwise velocity have the same frequency which implies that the unsteady flow does not behave as for high Reynolds numbers, where the fre quency of the drag is twice that of the velocity. As the Weissenberg number increases, the frequency increases quickly. Unfortunately, due to numerical instability, it is not possible to get results at high er Weissenberg numbers.

To illustrate that the flow is time periodic in the wake, we have plotted the phase portrait at several monitoring points in Fig. 24. Three cylinder diameters downstream, both the streamwise veloc ity and the transverse velocity oscillate with a significant ampli tude that increases as the Weissenberg number grows. The flow shows unsteadiness far downstream from the cylinder.

As Weissenberg number increases, the up down symmetry of the flow is broken by the polymer elasticity for $R e=40$ and $R e=100$ despite the fact that the problem is geometrically up down symmetric. This phenomenon does not happen for the New tonian fluid and low Weissenberg number flows. Fig. 25 shows the transverse velocity on the centerline. The mean transverse velocity is zero for the Newtonian fluid or for the viscoelastic fluid at small Weissenberg numbers. Beyond $W i=2$, the transverse velocity in creases near the cylinder as the Weissenberg number increases. Furthermore, the asymmetric transverse velocity grows as the Rey nolds number increases or the effect of viscosity decreases for low Reynolds number flows. In the same way, the conformation tensor component $\sigma_{x y}$ on the centerline is not symmetric when the Weiss enberg number is high, as shown in Fig. 26. The asymmetric part mainly appears in front and at the rear of the cylinder where the 


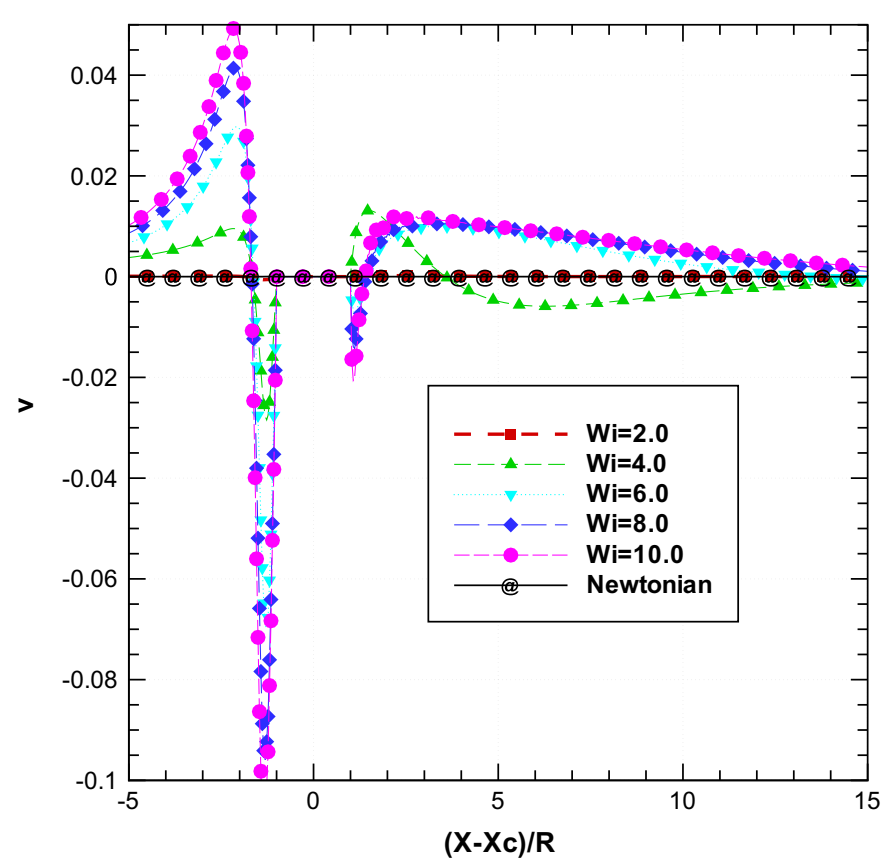

(a) $\operatorname{Re}=10$

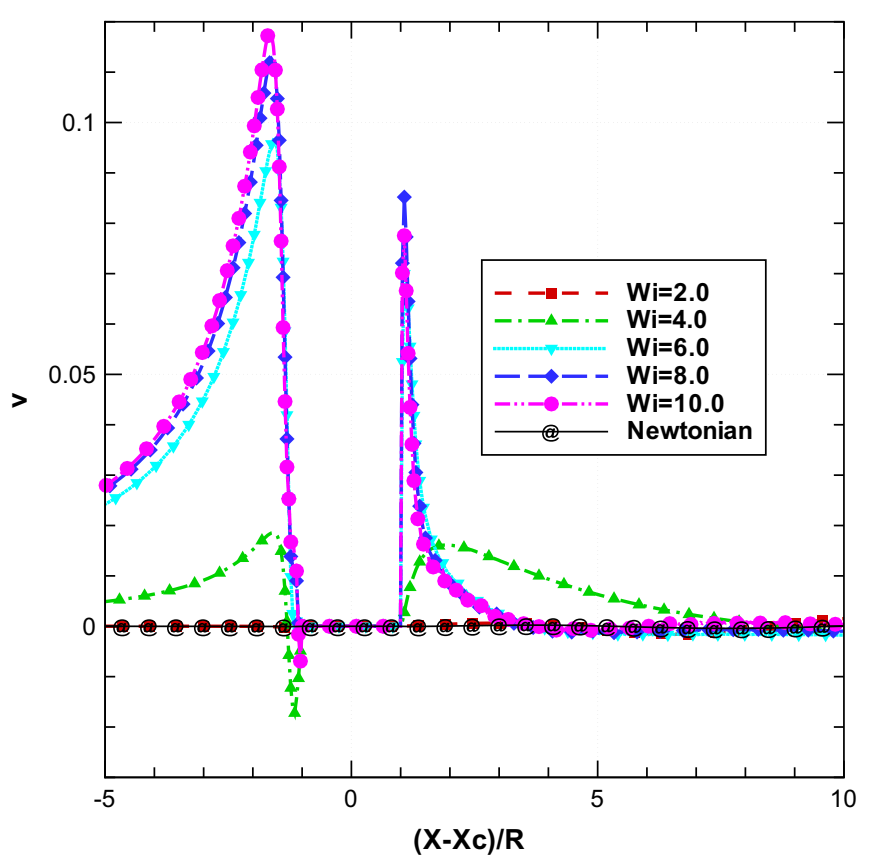

(b) $\operatorname{Re}=40$

Fig. 25. The mean transverse velocity on the centerline for $\epsilon=0.01$ and $B=0.1$.

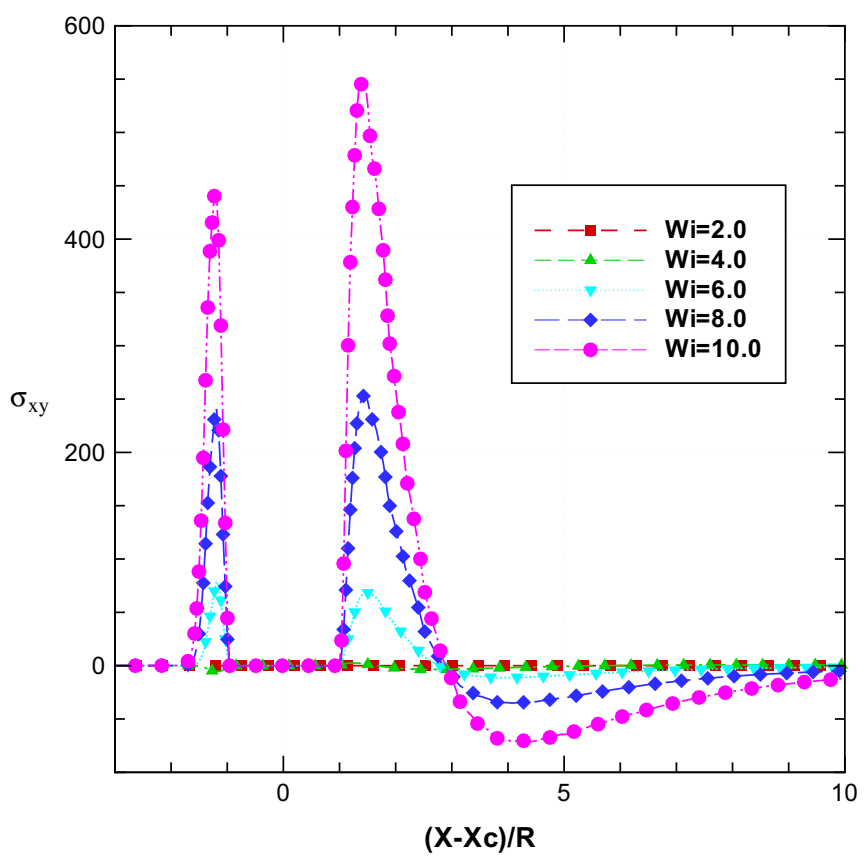

(a) $\operatorname{Re}=10$

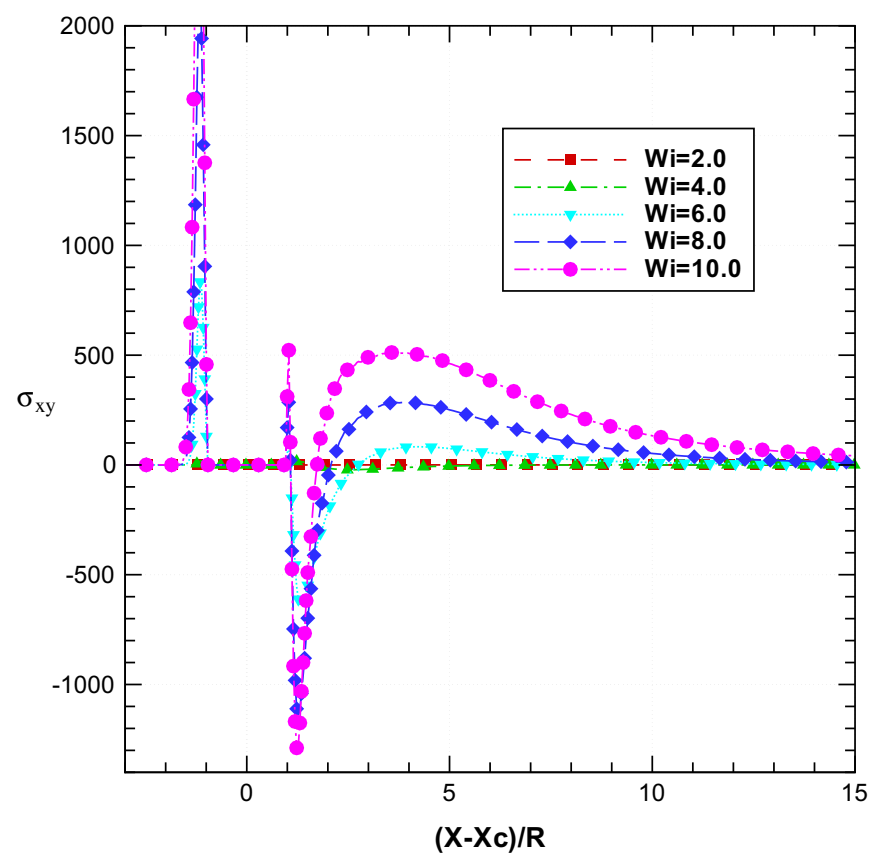

(b) $\operatorname{Re}=40$

Fig. 26. The conformation tensor component $\sigma_{x y}$ on the centerline for $\epsilon=0.01$ and $B=0.1$.

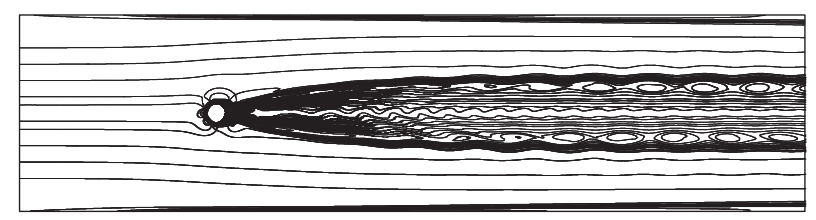

Fig. 27. An instantaneous vorticity field snapshot for $\epsilon=0.01$ and $B=0.1$ at $R e=3000$ and $W i=6.0$. elastic stress is large. The variation of $\sigma_{x y}$ along the centerline pro duces an elastic force, and the direction of the elastic force is nor mal to the centerline. In fact the value of $\sigma_{x y}$ is strongly coupled to the other components $\sigma_{x x}$ and $\sigma_{y y}$, they have a maximum value on both sides of the cylinder as we have reported, hence after a small disturbance, the shear may not be zero, it may produce a proper $\sigma_{x y}$ value, which can affect the balance and symmetry. Let us point out the up down symmetry is known to break down at Reynolds num bers between 10 and 40 from early experimental works [23] , even 


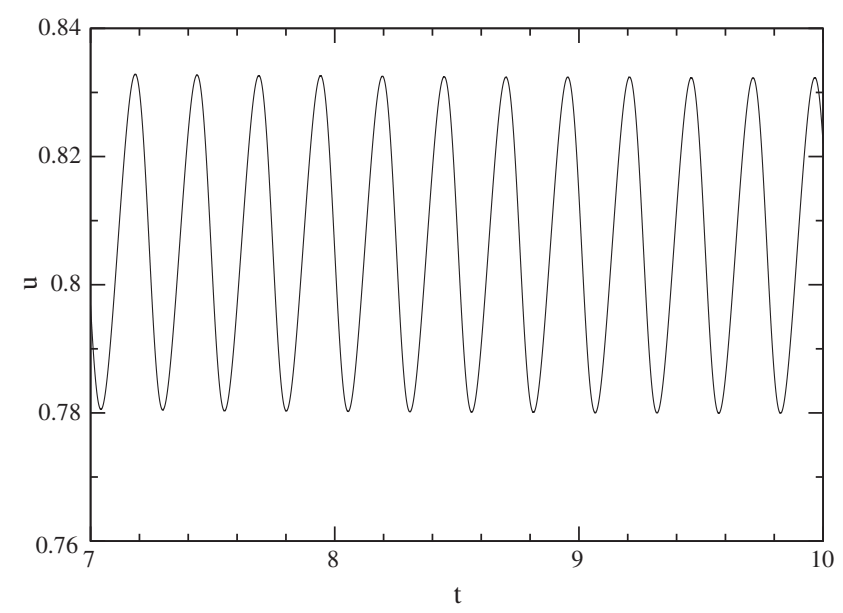

(a) streamwise velocity

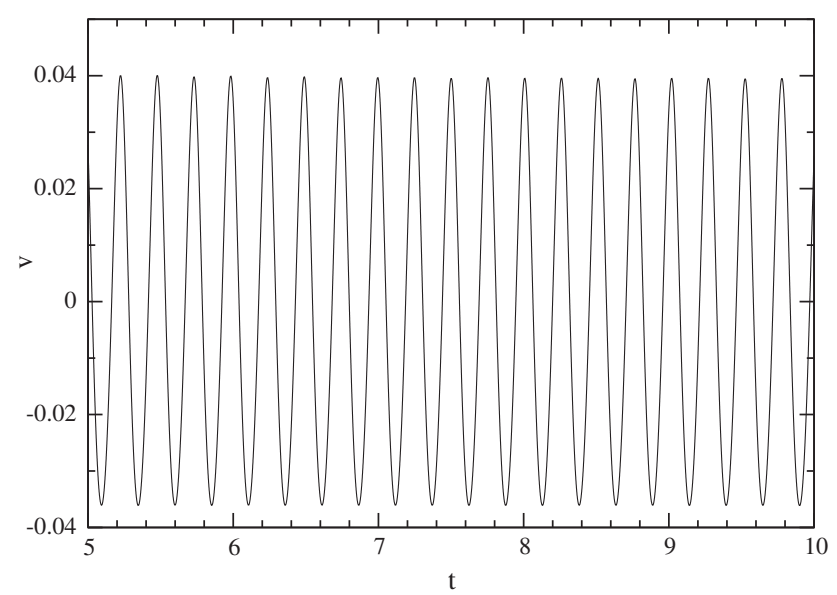

(b) transverse velocity

Fig. 28. The evolution of the velocity at the monitoring point $(2.3,0.4)$ for $\epsilon=0.01$ and $B=0.1$ at $R e=3000$ and $W i=6.0$.

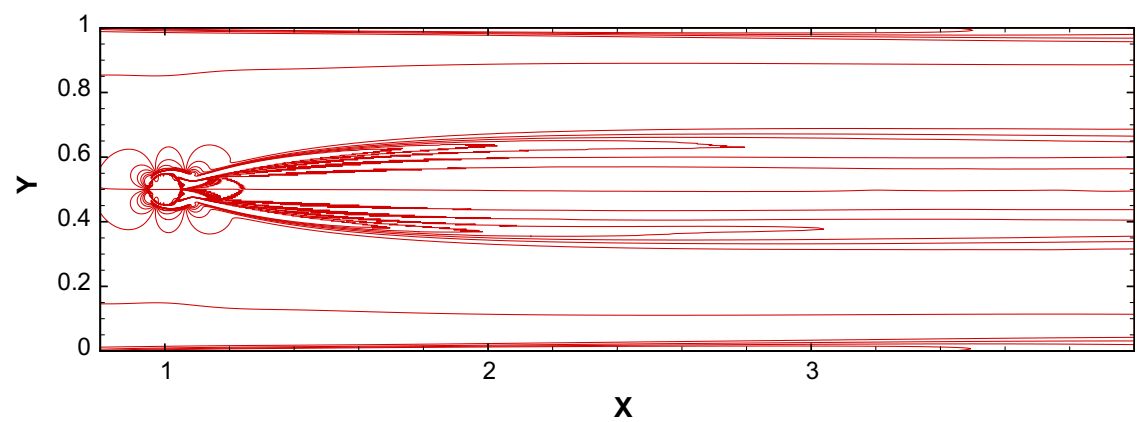

Fig. 29. The mean $d u / d y$ field at $R e=3000$ and $W i=6.0$ for $\epsilon=0.01$ and $B=0.1$.

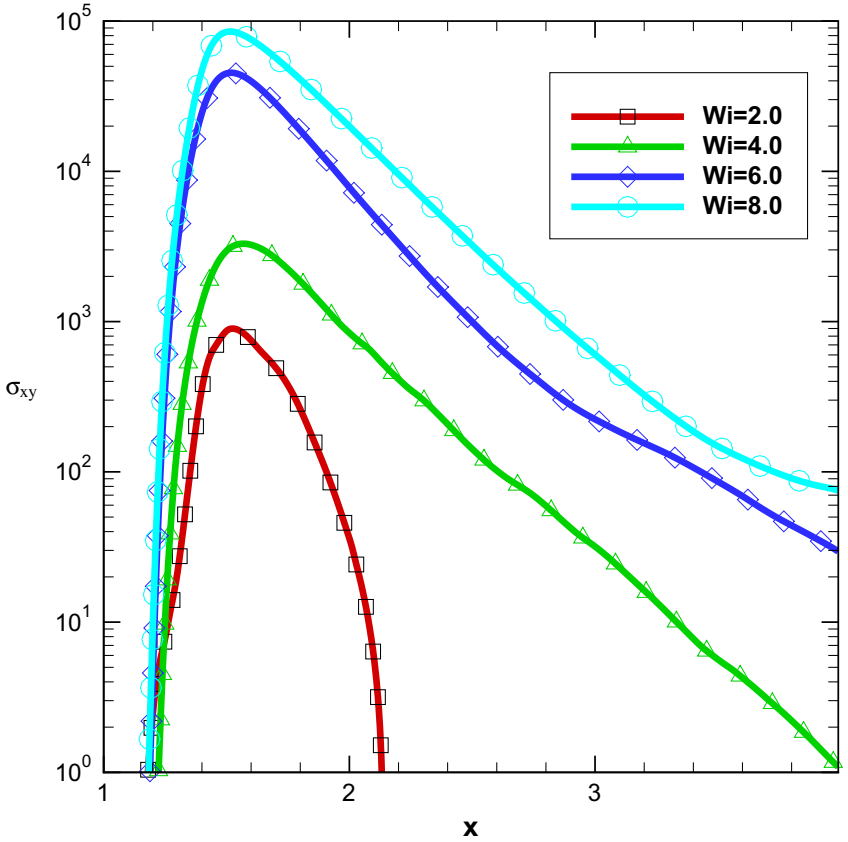

Fig. 30. The mean conformation tensor component $\sigma_{x y}$ at $y=0.6$ and $R e=3000$ for $\epsilon=0.01$ and $B=0.1$.

though it was not reported in that paper. We can observe the up down asymmetric streamlines on their flow visualization as the polymer concentration grows. This also indicates the up down symmetry could be broken by the high elasticity of the polymer.

As we have seen in Fig. 17, the Kelvin's 'cat's eye' pattern of the vorticity appears in the free shear layer at $R e=5000$ as the Weiss enberg number increases. This kind of instability also occurs at other Reynolds numbers when the Weissenberg number is high. As an example the flow at $R e=3000$ and $W i=6$ is plotted in Fig. 27. Fig. 28 shows the evolution of the velocity at the monitor ing point $(2.3,0.4)$. Both streamwise velocity and transverse veloc ity oscillate at the same frequency about 3.8, the transverse velocity having larger oscillating amplitude than the streamwise velocity.

In a homogeneous fluid, the instability can come from the high shear rate in the fluid. In the wake at high Weissenberg number, $d u / d y$ is the prominent contribution to the vorticity as shown in Fig. 29. The maximum shear rate in the wake at high Weissenberg number is 10 times bigger than in Newtonian fluid. However, the shear rate does not increase as the Weissenberg number grows be yond $W i_{2}$, as shown in Fig. 19 . Hence, the shear rate is not the only reason to induce instability in the viscoelastic wake flow. Indeed the shear rate at $W i=4$ is even larger than that at $W i=5$ when the Reynolds number is 5000 , but we have not observed the insta bility at $W i=4$, as shown in Fig. 17. The variation of the conforma tion tensor, especially $\sigma_{x y}$ making the fluids to swirl, may induce the instability. As we see in Fig. 30 , the mean $\sigma_{x y}$ at $y=0.6$ when $R e=3000$, increases as the Weissenberg number grows beyond $W i_{2}$. If the shear rate is not the direct reason of the instability, one may expect that a similar instability happens at lower Rey nolds numbers and higher Weissenberg numbers. However, it is 


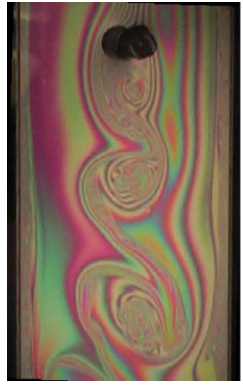

(a) $0 \mathrm{ppm}$

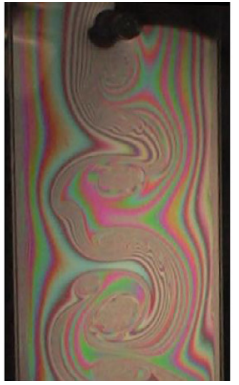

(b) $33 \mathrm{ppm}$

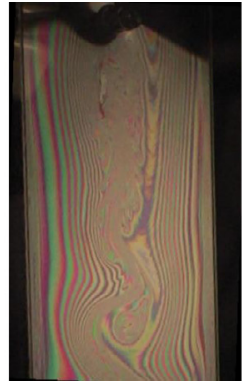

(c) $50 \mathrm{ppm}$

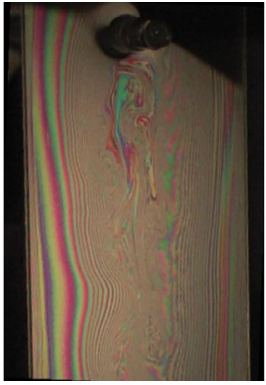

(d) $90 \mathrm{ppm}$

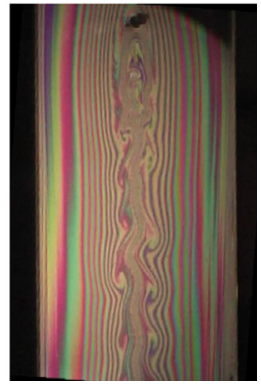

(e) $140 \mathrm{ppm}$

Fig. 31. Flow visualization of a two dimensional soap film flow over a cylinder at $R e \cong 2000$.

difficult to simulate viscoelastic flows at very high Weissenberg numbers. Thanks to the two dimensional soap film experiments $[28,29]$, we used different concentrations of long chain polymer molecules to achieve different Weissenberg numbers, as shown in Fig. 31 from soap film experiments at a Reynolds number about 2000. The maximum concentration $140 \mathrm{ppm}$ here corresponds to $W i \approx 20$. We can observe that vortex shedding disappears as the Weissenberg number increases, however a further increase of the concentration of polymer results in a flow instability for the cylin der wake.

\section{Conclusions}

External flows past obstacles, such as a circular cylinder, have been studied extensively because they are rich in flow phenomena and practical applications. The addition of polymers has an impor tant effect on such flows and can therefore be useful to control the flow in a passive way. We have performed two dimensional numerical simulations of flow past a circular cylinder in a channel for Newtonian fluid and dilute polymer solutions in a wide param eter range. Comparisons and coincident results with other numer ical results and experiments show that our flow solver with a simple Oldroyd B model can capture the main flow behaviors with results that are in agreement with previous results and observa tions available in the literature.

Our numerical simulations show that a small amount of poly mer additive may increase or decrease the drag of the cylinder. We used a phase diagram in $R e$ Wi space to capture the drag behavior at a single blockage $B=0.1$. Whether the Reynolds num ber corresponds to an unsteady wake or steady wake for the New tontion fluid is crucial to the drag behavior of the cylinder in the presence of polymers. When the Reynolds number is smaller than the transitional Reynolds number, the drag of the cylinder and the flow are different from the Newtonian flow when the Weissenberg number exceeds a critical value: The drag increases as the Weiss enberg number increases in this case. On the other hand, there are two threshold Weissenberg numbers to sort out the three kinds of drag behavior (Newtonian drag, drag reduction and drag enhancement) when the Newtonian wake is unsteady. The corre sponding main flow features change as the drag regime changes. This behavior can be rationalized using two threshold Weissenberg numbers. In the drag reduction regime, the mean wake length and the shedding period are increased, and the polymer also sup presses the time oscillations in the drag and lift forces. The drag enhancement regime at large Reynolds numbers is accompanied by a total suppression of the vortex shedding instability, the pres ence of large elongation rates and a large pressure well at the rear of the cylinder. As for the lift force on the cylinder, it decreases in most cases when the polymers affect the flow mainly when the vortex shedding of the unsteady wake is inhibited. However, the lift may increase when the elasticity destroys the flow symmetry at high Weissenberg numbers. As the Weissenberg number in creases, numerical simulations and soap film experiments show that the viscoelasticity of the solution may result in flow instabilities.

\section{Acknowledgment}

The authors would like to thank Prof. Oliveira for providing the experimental results in [51].

\section{References}

[1] Y. Amarouchene, H. Kellay, Polymers in 2D turbulence: suppression of large scale fluctuation, Phys. Rev. Lett. 89 (2002) 104502.

[2] P. Angot, C.H. Bruneau, P. Fabrie, A penalization method to take into account obstacles in an incompressible flow, Numer. Math. 81 (1999) 497-520.

[3] F.P.T. Baaijens, S.H.A. Selen, H.P.W. Baaijens, G.W.M. Peters, H.E.H. Meijer, Viscoelastic flow past a confined cylinder of a low density polyethylene melt, J. Non-Newton. Fluid Mech. 68 (1997) 173-203.

[4] C.H. Bruneau, P. Fabrie, New efficient boundary conditions for incompressible Navier-Stokes equations: a well-posedness result, M2AN 30 (1996).

[5] C.H. Bruneau, O. Greffier, H. Kellay, Numerical study of grid turbulence in two dimensions and comparison with experiments on turbulent soap films, Phys. Rev. E 60 (1999) R1165.

[6] C.H. Bruneau, H. Kellay, Experiments and direct numerical simulations of twodimensional turbulence, Phys. Rev. E 71 (2005) 046305.

[7] C.H. Bruneau, M. Saad, The 2D lid-driven cavity problem revisited, Comput. Fluids 35 (2006) 326-348.

[8] O. Cadot, S. Kumar, Experimental characterization of viscoelastic effects on two- and three-dimensional shear instabilities, J. Fluid Mech. 416 (2000) 151172.

[9] O. Cadot, M. Lebey, Shear instability inhibition in a cylinder wake by local injection of a viscoelastic fluid, Phys. Fluids 11 (1999) 494-496.

[10] A.E. Caola, Y.L. Joo, R.C. Armstrong, R.A. Brown, Highly parallel time integration of viscoelastic flows, J. Non-Newton. Fluid Mech. 100 (2001) 191-216.

[11] M.D. Chilcott, J.M. Rallison, Creeping flow of dilute polymer solutions past cylinders and spheres, J. Non-Newton. Fluid Mech. 29 (1988) 381-432.

[12] Y.J. Choi, M.A. Hulsen, H.E.H. Meijer, Simulation of the flow of a viscoelastic fluid around a stationary cylinder using an extended finite element method, Comput. Fluids 57 (2012) 183-194.

[13] J.R. Cressman, Q. Bailey, W.I. Goldburg, Modification of a vortex street by a polymer additive, Phys. Fluids 13 (2001) 867-871.

[14] H.S. Dou, N. Phan-Thien, The flow of an Oldroyd-B fluid past a cylinder in a channel: adaptive viscosity vorticity formulation, J. Non-Newton. Fluid Mech. 87 (1999) 47-73.

[15] Y.R. Fan, R.I. Tanner, N. Phan-Thien, Galerkin/least-squares finite-element methods for steady viscoelastic flows, J. Non-Newton. Fluid Mech. 84 (1999) 233-256.

[16] N. Francois, D. Lasne, Y. Amarouchene, B. Lounis, H. Kellay, Drag enhancement with polymers, Phys. Rev. Lett. 100 (2008) 018302.

[17] A. Groisman, V. Steinberg, Elastic turbulence in a polymer solution flow, Nature 405 (2000) 53-55.

[18] A. Groisman, V. Steinberg, Elastic turbulence in curvilinear flows of polymer solutions, New J. Phys. 6 (2004) 29.

[19] P.Y. Huang, J. Feng, Wall effects on the flow of viscoelastic fluids around a circular cylinder, J. Non-Newton. Fluid Mech. 60 (1995) 179-198.

[20] X. Huang, N. Phan-Thien, R.I. Tanner, Viscoelastic flow between eccentric rotating cylinders: unstructured control volume method, J. Non-Newton. Fluid Mech. 64 (1996) 71-92. 
[21] M.A. Hulsen, R. Fattal, R. Kupferman, Flow of viscoelastic fluids past a cylinder at high Weissenberg number: stabilized simulations using matrix logarithms, J. Non-Newton. Fluid Mech. 127 (2005) 27-39.

[22] D.F. James, Boger fluids, Annu. Rev. Fluid Mech. 41 (2009) 129-142.

[23] D.F. James, A.J. Acosta, The laminar flow of dilute polymer solutions around circular cylinders, J. Fluid Mech. 42 (1970) 269-288.

[24] H. Kato, Y. Mizuno, An experimental investigation of viscoelatic flow past a circular cylinder, Bull. JSME 36 (1983) 529-536.

[25] H. Kellay, Polymers suppress the inverse transfers of energy and the enstrophy flux fluctuations in two-dimensional turbulence, Phys. Rev. E 70 (2004) 036310.

[26] H. Kellay, C.H. Bruneau, X.L. Wu, Probability density functions of the enstrophy flux in two dimensional grid turbulence, Phys. Rev. Lett. 84 (2000) 1696-1699.

[27] H. Kellay, W. Goldburg, Two-dimensional turbulence: a review of some recent experiments, Rep. Prog. Phys. 65 (2002) 845-894.

[28] H. Kellay, X.L. Wu, W. Goldburg, Experiments with turbulent soap films, Phys. Rev. Lett. 74 (1995) 3975-3978.

[29] H. Kellay, X.L. Wu, W. Goldburg, Vorticity measurements in turbulent soap films, Phys. Rev. Lett. 80 (1998) 277-280.

[30] A.W. Liu, D.E. Bornside, R.C. Armstrong, R.A. Brown, Viscoelastic flow of polymer solutions around a periodic, linear array of cylinders: comparisons of predictions for microstructure and flow fields, J. Non-Newton. Fluid Mech. 77 (1998) 153-190.

[31] J.L. Lumley, Drag reduction by additives, Annu. Rev. Fluid Mech. 1 (1969) 367384.

[32] X. Ma, V. Symeonidis, G.E. Karniadakis, A spectral vanishing viscosity method for stabilizing viscoelastic flows, J. Non-Newton. Fluid Mech. 115 (2003) 125155.

[33] J.H. McKinley, R.C. Armstrong, R.A. Brown, The wake instability in viscoelastic flow past confined circular cylinders, Philos. Trans. R. Soc. Lond. A344 (1993) 265-304.

[34] T. Min, H. Choi, J.Y. Yoo, Maximum drag reduction in a turbulent channel flow by polymer additives, J. Fluid Mech. 492 (2003) 91-100.

[35] E. Mitsoulis, Numerical simulation of confined flow of polyethylene melts around a cylinder in a planar channel, J. Non-Newton. Fluid Mech. 76 (1998) 327-350.

[36] T. Odijk, Polymer-induced vortex modification in decaying two-dimensional turbulence, Physica A 258 (1998) 329-340.

[37] J.G. Oldroyd, On the formulation of rheological equations of state, Proc. R. Soc. Lond. A 200 (1950) 523-541.

[38] P.J. Oliveira, A.I.P. Miranda, A numerical study of steady and unsteady viscoelastic flow past bounded cylinders, J. Non-Newton. Fluid Mech. 127 (2005) 51-66.
[39] P.J. Oliveira, Method for time-dependent simulations of viscoelastic flows: vortex shedding behind cylinder, J. Non-Newton. Fluid Mech. 101 (2001) 113137.

[40] R.G. Owens, C. Chauviere, T.N. Philips, A locally-upwinded spectral technique (LUST) for viscoelastic flows, J. Non-Newton. Fluid Mech. 108 (2002) 49-71.

[41] C.J. Pipe, P.A. Monkewtiz, Vortex shedding in flows of dilute polymer solutions, J. Non-Newton. Fluid Mech. 139 (2006) 4-67.

[42] D. Richter, G. Iaccarino, E.S.G. Shaqfeh, Simulations of three-dimensional viscoelastic flows past a circular cylinder at moderate Reynolds numbers, J. Fluid Mech. 651 (2010) 415-442.

[43] D. Richter, G. Iaccarino, E.S.G. Shaqfeh, Effects of viscoelasticity in the high Reynolds number cylinder wake, J. Fluid Mech. 693 (2012) 297-318.

[44] M. Sahin, R.G. Owens, On the effects of viscoelasticity on two-dimensional vortex dynamics in the cylinder wake, J. Non-Newton. Fluid Mech. 123 (2004) 121-139.

[45] A.H. Shiang, A. Öztekin, J.C. Lin, D. Rockwell, Hydroelastic instabilities in viscoelastic flow past a cylinder confined in a channel, Exp. Fluids 28 (2000) $128-142$.

[46] S.P. Singh, S. Mittal, Flow past a cylinder: shear layer instability and drag crisis, Int. J. Numer. Meth. Fluids 47 (2005) 75-98.

[47] J. Sun, M.D. Smith, R.C. Armstrong, R.A. Brown, Finite element method for viscoelastic flows based on the discrete adaptive viscoelastic stress splitting and the discontinuous Galerkin method: DAVSS-G/DG, J. Non-Newton. Fluid Mech. 86 (1999) 281-307.

[48] R. Sureshkumar, A.N. Beris, Effect of artificial stress diffusivity on the stability of numerical calculations and the flow dynamics of time-dependent viscoelastic flows, J. Non-Newton. Fluid Mech. 60 (1995) 53-80.

[49] B.A. Toms, Some observation on the flow of linear polymer solutions through straight tubes at large Reynolds number, Proceedings of the First International Congress of Rheology, vol. 2, North Holland, Amsterdam, 1949, pp. 135-141.

[50] P.S. Virk, Drag reduction in rough pipes, J. Fluid Mech. 45 (1970) 225-246.

[51] J.M. Verhelst, F.T.M. Nieuwstadt, Visco-elastic flow past circular cylinders mounted in a channel: experimental measurements of velocity and drag, J. Non-Newton. Fluid Mech. 116 (2004) 301-328.

[52] C.H.K. Williamson, Vortex dynamics in the cylinder wake, Annu. Rev. Fluid Mech. 28 (1996) 477-539.

[53] Y.L. Xiong, C.H. Bruneau, H. Kellay, Drag enhancement and drag reduction in viscoelastic fluid flow around a cylinder, Europhys. Lett. 91 (2010) 640001.

[54] Y.L. Xiong, C.H. Bruneau, H. Kellay, Direct numerical simulations of 2D channel flows in the presence of polymers, Europhys. Lett. 95 (2011) 640003. 